\title{
Measuring Affect Dynamics: An Empirical Framework
}

\author{
Sergio Pirla* \\ Universitat Pompeu Fabra, Spain \\ Maxime Taquet* \\ University of Oxford, United Kingdom \\ Jordi Quoidbach \\ Universitat Ramon Llull, ESADE, Spain
}

Authors' note

*These authors contributed equally. Corresponding author: Sergio Pirla (sergio.pirla@upf.edu). 


\begin{abstract}
A fast-growing body of evidence from experience sampling studies suggests that affect dynamics are associated with well-being and psychological health. But heterogeneity in experience sampling approaches impedes reproducibility and scientific progress. Leveraging a large dataset of 7016 individuals, each providing over 50 affect reports, we introduce an empirically-derived framework to help researchers design well-powered and efficient experience sampling studies. Our research reveals three general principles. First, a sample of 200 participants and 20 observations per person yields sufficient power to detect medium size associations for most affect dynamic measures. Second, for trait and time-independent variability measures of affect (e.g., S.D.), distant sampling study designs (i.e., a few daily measurements spread out over several weeks) leads to more accurate estimates than close sampling study designs (i.e., several daily measurements concentrated over a few days), whereas differences in accuracy across sampling methods were inconsistent and of little practical significance for temporally dependent affect dynamic measures (i.e., RMSSD, autocorrelation coefficient, TKEO, and PAC). Third, across all affect dynamics measures, sampling exclusively on specific days or time windows leads to little to no improvement over sampling at random times. Because the ideal sampling approach varies for each affect dynamics measure, we provide a companion R-package, an online calculator, and a series of benchmark effect sizes to help researchers address three fundamental how's of experiencesampling: How many participants to recruit? How often to solicit them? And for how long?
\end{abstract}




\section{Introduction}

With the advent of mobile phones, the experience sampling method (ESM;

Csikszentmihalyi \& Larson, 1984; also known as ecological momentary assessment; Stone \& Shiffman, 1994) has quickly become the gold standard to study human emotion (Stone et al., 1998; Lucas, Wallsworth, Anusic, \& Donnellan, 2021). Rather than relying on retrospective reports ("How did you feel yesterday?") or cross-sectional surveys (“How do you feel in general?”), researchers in psychology, psychiatry, and behavioral science are now routinely capturing people's subjective experience in the moment through short mobile questionnaires. Experience-sampling not only alleviates recall and evaluative bias (Fredrickson \& Kahneman, 1993; Redelmeier \& Kahneman, 1996; Schimmack \& Oishi, 2005), but also allows to uncover how dynamic aspects of people's emotional lives (e.g., fluctuation, inertia) play a crucial role in mental and physical health (for a meta-analysis, see Houben, Van Den Noortgate, \& Kuppens, 2015).

Since the first ESM studies in the 70s, countless articles have discussed the promises of the method to study emotion (Ellison et al., 2020; Fisher \& To, 2012; Myin-Germeys et al., 2018; Schimmack, 2003; Scollon, Prieto, \& Diener, 2009), and many technical solutions have blossomed (see Arslan, Walther, \& Tata, 2020 and Meers et al., 2020 for overviews). However, scientists have been astonishingly left to their own devices when it comes to conducting such research. Imagine, for example, that you want to assess how happy a person feels. How many moments of their daily life should you observe to capture their average happiness accurately? What about their propensity to experience mood swings? How spread or concentrated should your observations be? These questions are critical to the design of well-powered, cost-efficient ESM studies in affective sciences. However, an abysmal $2 \%$ of emotion ESM studies justify their sampling procedure (Trull \& Ebner-Priemer, 2020), leading to important power, reproducibility, and suboptimal resource-allocation issues (e.g., 
Aguinis, Gottfredson, \& Culpepper, 2013; Calamia 2019; Kirtley, Lafit, Achterhof, Hiekkaranta, \& Myin-Germeys, 2019).

In what follows, we first provide a brief overview of the experience-sampling method in emotion research and the primary individual differences studied through this method. We then review the wide variety of sampling practices used to capture these individual differences. Finally, we stress the importance of relying on actual data to make critical decisions about how many participants to recruit and how often, when, and how long to observe them.

\section{Experience Sampling and Affective Sciences}

Experience sampling involves repeated measurement of people's experiences, as it unfolds in real-time in their everyday lives (Conner, Tennen, Fleeson, \& Barrett, 2009). It offers several advantages over traditional lab or survey-based emotion research.

First, by capturing emotions as they naturally occur in everyday life - rather than relying on artificial laboratory manipulation - ESM helps uncover how complex, intertwined, and diverse our affective reactions truly are (e.g., Dejonckheere et al., 2018; Kerr et al., 2021). For example, while theorists have debated the idea that people can experience two oppositely valenced emotions for decades, results from experience-sampling suggest that this is a ubiquitous experience in everyday life: People report experiencing mixed emotions about a third of the time (Trampe, Quoidbach, \& Taquet, 2015).

Second, by capturing emotions in real-time, ESM reduces recall and evaluative biases (e.g., Solhan, Trull, Jahng, \& Wood, 2009; Stone et al., 1998). For example, people's retrospective ratings of how they felt during emotional experiences are overly influenced by these experiences' last and most intense moments (Fredrickson \& Kahneman, 1993; Kahneman, Fredrickson, Schreiber, \& Redelmeier, 1993; Redelmeier \& Kahneman, 1996). Similarly, global reports of affective states can be tainted by aspects of one's life that happen 
to be salient at the moment (see Schimmack \& Oishi, 2005, for a meta-analysis)—for example, asking people questions about politics right before asking them how happy they feel overall substantially reduces happiness scores (Deaton \& Stone, 2016).

Third, by capturing emotions on multiple occasions, ESM allows studying the role of changing contexts on people's emotions. For example, researchers have been able to quantify what type of daily activities (Choi et al., 2016; Taquet et al., 2016) or social interaction partners (Quoidbach et al., 2019) impact people’s momentary happiness. For instance, Mueller and colleagues (2019) examined over 50,000 episodes of social interactions. They found that social (vs. task-oriented) conversations with close (vs. less close) others were associated with higher momentary happiness.

\section{Experience Sampling and Affect dynamics Measures}

Beyond increased ecological validity and accuracy, a major contribution of ESM is that it allows to uncover how individual differences in affect dynamics, that is, trajectories, patterns, and regularities in people's emotion over time, play a critical role in mental health and psychopathology (Kuppens, 2015; Kuppens \& Verduyn, 2017). Dozens of new affect dynamics measures have been introduced over the past decade, each designed to evaluate a unique aspect of people's emotional lives. Whereas the incremental validity of several of these indicators is currently debated (Dejonckheere et al., 2019; Lapate \& Heller, 2020; Wendt et al., 2020), the most common measures of affect dynamics in the literature include trait affect, affect variability, affect instability, and affect inertia (see Table 1).

Trait affect represents people's propensity to experience negative or positive affect and is considered a relatively stable personality characteristic (e.g., Watson \& Tellegen, 1985). It is typically captured as the individual mean of affective states. Affect variability represents whether people's affective state tends to fluctuate over time, regardless of when these fluctuations occur. It is typically operationalized as the intraindividual standard 
deviation in affective states (Nesselroade \& Salthouse, 2004; Ram \& Gerstorf, 2009) or a mean-corrected version of this intraindividual standard deviation that avoids confounding effects of the mean (Mestdagh et al., 2018). In contrast, affect instability is a function of temporal order and represents whether people's affective states tend to change abruptly from one moment to the next. Across different research domains, instability has been typically measured as the root mean square of successive differences (RMSSD; Jahng, Wood, \& Trull, 2008), the probability of acute change (PAC; Trull et al., 2008), or the Teager-Kaiser Energy Operator (TKEO; Solnik et al., 2010; Tsanas et al., 2016). Finally, affect inertia represents the degree to which people's affective states persist from one moment to the next. It is typically captured as an autoregressive correlation between an individual's current affective state and their previous affective state in time series (AR; e.g., Kuppens, Allen, \& Sheeber, 2010). 


\begin{tabular}{|c|c|c|c|}
\hline Measure & Index & Formal Definition & Interpretation \\
\hline Trait & Average & $\frac{\sum x_{i}}{n}$ & Average affect. \\
\hline Variability & $\begin{array}{l}\text { Standard Deviation } \\
\text { (SD) }\end{array}$ & $\frac{\sum\left(x_{i}-M\right)^{2}}{n}$ & $\begin{array}{l}\text { Standard deviation of } \\
\text { affect. }\end{array}$ \\
\hline Variability & $\begin{array}{l}\text { Relative Standard } \\
\text { Deviation (Rel. SD) }\end{array}$ & $\frac{S D}{\max (S D \mid M)}$ & $\begin{array}{l}\text { Mean-corrected estimate } \\
\text { of the standard deviation } \\
\text { for bounded variables } \\
\text { (Mestdagh et al. 2018). }\end{array}$ \\
\hline Instability & $\begin{array}{l}\text { Root Mean Square } \\
\text { of Successive } \\
\text { Differences } \\
\text { (RMSSD) }\end{array}$ & $\sqrt{\frac{\sum\left(x_{i}-x_{i+1}\right)^{2}}{n-1}}$ & $\begin{array}{l}\text { Average change across } \\
\text { successive affect } \\
\text { observations. }\end{array}$ \\
\hline Instability & $\begin{array}{l}\text { Teager-Kaiser } \\
\text { Energy Operator } \\
\text { (TKEO) }\end{array}$ & $\frac{\sum\left(x_{i}^{2}-x_{i-1} \cdot x_{i+1}\right)}{n-2}$ & $\begin{array}{l}\text { Measure of change across } \\
3 \text { affect reports. Useful in } \\
\text { identifying mood spikes. }\end{array}$ \\
\hline Instability & $\begin{array}{l}\text { Probability of } \\
\text { Acute Change } \\
\text { (PAC) }\end{array}$ & $\frac{\sum I\left(x_{i+1}-x_{i}, d_{0.9}\right)}{n-1}$ & $\begin{array}{l}\text { Likelihood of extreme } \\
\text { affect changes. }\end{array}$ \\
\hline Inertia & $\begin{array}{l}\text { Auto-correlation } \\
\text { Coefficient }\end{array}$ & $\frac{\sum\left(x_{i}-M\right)\left(x_{i+1}-M\right)}{\sum\left(x_{i}-M\right)^{2}}$ & $\begin{array}{l}\text { Correlation between } \\
\text { successive affect reports. }\end{array}$ \\
\hline
\end{tabular}

Table 1: Affect dynamics measures included in our study. In the formulas, $x_{i}$ stands for the $i^{\text {th }}$ current affect report of a given individual. Similarly, $n$ represents the total number of observations collected for the individual. $S D$ and $M$ represent respectively the standard deviation and mean affect reported by a given individual. Finally, $I\left(x_{i+1}-x_{i}, d_{0.9}\right)$ defines a binary variable taking a value of 1 if $\left(x_{i+1}-x_{i}\right)$ is greater than $d_{0.9}$ in absolute terms and 0 otherwise, where $d_{0.9}$ represents the $90^{\text {th }}$ percentile in the distribution of absolute affect changes across all participants in the sample.

Accumulating empirical evidence shows that affect dynamics are associated with well-being and psychological health. For example, research shows strong associations between average affect and depression (Golier et al., 2001, Thompson et al., 2012), post- 
traumatic stress disorder (Golier et al., 2001), borderline personality disorder (Zeigler-Hill et al., 2006), and anxiety disorders (Bowen et al., 2006). Likewise, affect variability predicts lower subjective well-being (Gruber, Kogan, Quoidbach, \& Mauss, 2013) and affective disorders (Bowen et al., 2004; Golier et al., 2001; McConville \& Cooper, 1996). Affect instability is linked to poor mental health and several psychological disorders, including anxiety (Pfaltz et al., 2010), bipolar disorder (Jones et al., 2005), borderline personality disorder (Ebner-Priemer et al., 2007, Santangelo et al., 2014), major depressive disorder (Aan het Rot et al., 2012), and bulimia nervosa (Anestis et al., 2010). Finally, affect inertia is related to low self-esteem, neuroticism, and trait rumination (see Trull et al., 2015, for a review).

\section{Affect dynamics Measures: The Wild West of Sampling Approaches}

The field of affect dynamics holds great promise. But the wide range of outcomes that have been related to affect dynamics measures is met by an even wider range of methodological approaches to study them. We examined the sampling characteristics of 423 ambulatory assessment studies of affect included in five major review articles (Aan het Rot et al., 2012; Dunster et al., 2021; Ebner-Priemer \& Trull, 2009; Houben et al., 2015; MyinGermeys et al., 2009). Of these, 88 studies estimated at least one core affect dynamics measure. Our examination revealed a wide range of practices with samples ranging from 10 to 500 individuals and 14 to over 400 observations per individual (see Figure 1). Studies also crucially differed with regard to when and for how long they surveyed participants. Some studies favored close sampling - many daily questionnaires collected over a short period (e.g., ten questionnaires a day for a week; Delespaul \& DeVries, 1987; Myin-Germeys et al., 2000; Peeters et al., 2010) - whereas others favored distant sampling — few daily questionnaires collected over a longer period (e.g., two questionnaires a day for two weeks; Chepenik et al., 2006; Links et al., 2003). Some studies systematically sent questionnaires on 
specific days (weekdays vs. weekends; Beal \& Ghandour, 2011) or at specific times (e.g., morning, afternoon, or evenings; Gruber et al., 2013; Knowles et al., 2007; Links et al., 2003; Zeigler-Hill \& Abraham, 2006), while other studies probed participants at random times (Havermans et al., 2007; Peeters et al., 2006; Trull et al., 2008).

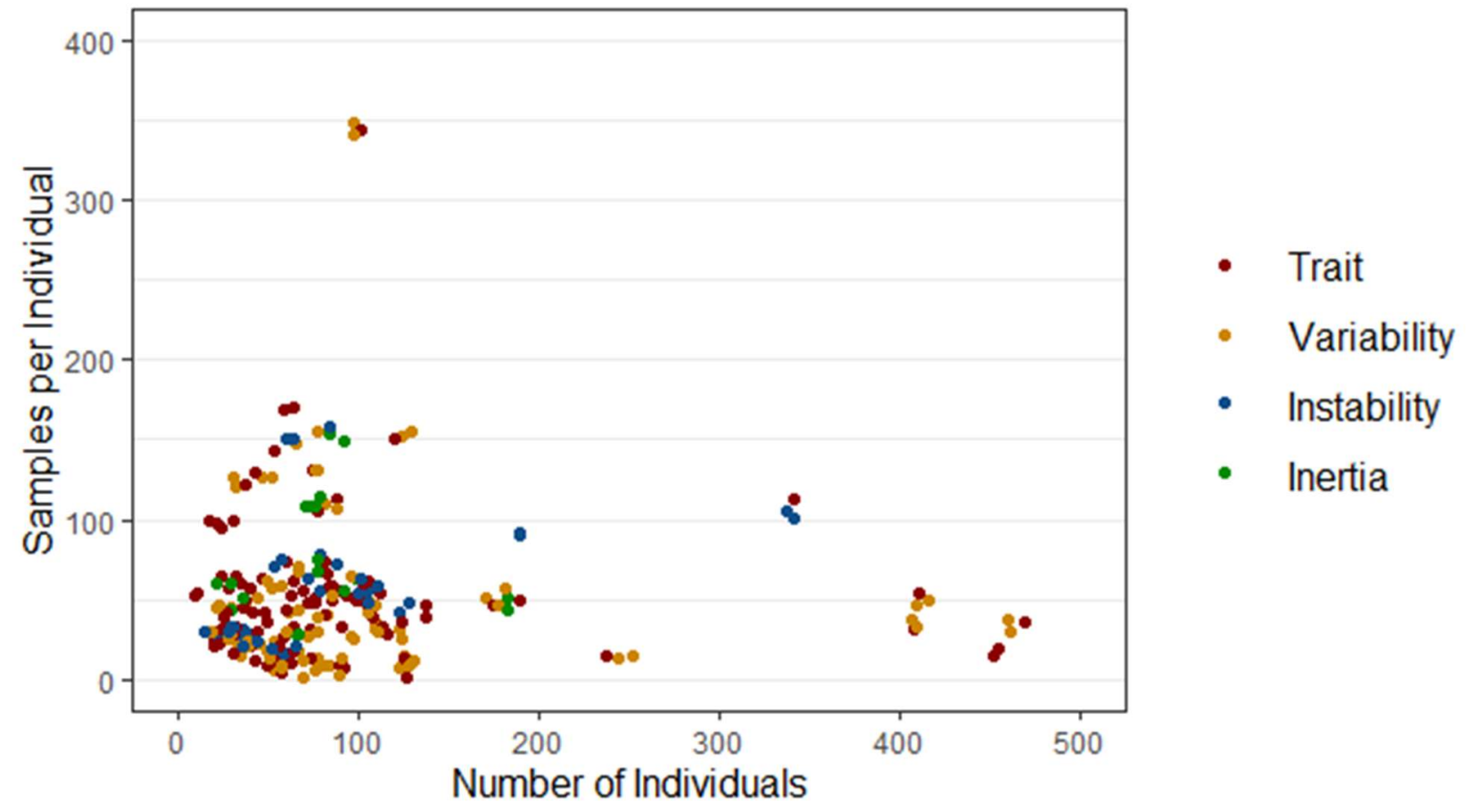

Figure 1. Distribution of the number of individuals sampled and the number of observations per individual in 88 emotion ESM studies.

The lack of a standardized approach has profound ramifications. First, it leads researchers to rely on heuristics, opportunities, or unfounded conventions to define their sample size, rather than rely on adequate power calculation. For example, a common design in the ESM literature (around 40\% of the studies) is to collect observations ten times a day for six consecutive days, even if this approach is neither based on power considerations nor necessarily optimal (Myin-Germeys, 2018). The current lack of evidence to guide sampling decisions might result in underpowered studies, leading to missed opportunities to discover true effects and inflated effect sizes of discovered effects (Ioannidis, 2008). Combined with publication bias and the difficulty to publish null results, underpowered studies are a root 
cause of the dire claim that most research findings are false (Ioannidis, 2005). Whereas underpowered studies are of great concern, researchers should not find solace in overpowered studies. Recruiting more participants than is needed or running a study for longer than necessary puts an unnecessary burden on human subjects, increases the risk of attrition, and misallocates essential resources. It might also be unethical if the answer to the research question at hand can improve people's health or quality of life.

\section{Developing an Empirical Framework}

A validated framework for study design would considerably advance the study of affect dynamics. But this framework needs to be determined on real affect data and not abstract or simulated power calculations (Arend \& Schäfer, 2019; Astivia et al., 2019; Lane \& Hennes, 2018). This is because the data generation process is complex and cannot be accurately captured by parametric models. Affect time series are stochastic processes that depend, in nonlinear ways, on various intertwined variables (e.g., time, weather, social interactions, cortisol level, physical wellness), many of which cannot be measured. Moreover, affect dynamics measures (e.g., the root mean squared successive differences) are themselves nonlinear summary statistics derived from these time series. Therefore, any valid framework to designing affect dynamics studies needs to link the probability distribution of these nonlinear transformations of non-uniformly sampled stochastic time series to the sampling process. In practice, this is most readily achieved using real data and assessing power empirically.

To address these issues, we build on a large dataset of 7016 individuals, each providing over 50 affect reports at random moments using smartphones. We first analyze how many samples are needed to capture a person's "true" affect dynamics in terms of trait affect (i.e., average), affect variability (i.e., within-person standard deviation), affect instability (i.e., RMSSD, TKEO, and PAC), and affect inertia (i.e., autocorrelation). We then 
investigate how strategic considerations in terms of timing between samples, time of the day, and days of the week change the number of samples needed to accurately capture these affect dynamics measures. Finally, we examine how the power to detect an association between the different measures and a given outcome varies as a function of sampling procedures. In doing so, we provide researchers with an easy-to-use companion R-package and an online calculator to address three fundamental how's of experience sampling studies: How many participants to recruit? How often to solicit them? And for how long?

\section{Method}

\section{Participants and Experience Sampling}

We collected our data using "58 seconds," a free francophone smartphone application designed to assess different aspects of people's well-being by sending short questionnaires at random times of the day. Participants provided basic information on age, gender, and country of residence at sign-up (see Note 1 of Supplementary Materials). They were then asked to select which days of the week, within what time windows, and how many sample requests they wanted to receive (default $=4$ daily questionnaires between 9 a.m. and 10 p.m. each day of the week). We ensured random sampling through a notification system that did not require users to be connected to the internet. Each questionnaire consisted of 4-6 questions selected from an extensive battery of items. The sample and item pool has been extensively described in other publications (Taquet et al., 2020; Quoidbach, Taquet, Desseilles, de Montjoye, \& Gross, 2019). For the purpose of this study, we focus on participants who reported their current affective state (using a slider from 0-very unhappy to 100-very happy) at least 50 times. This subsample includes 7016 individuals $\left(M_{\text {Age }}=29.9\right.$, S.D. Age $=9.9 ; 74 \%$ female $)$ who each provided an average of $111.6(S . D .=87.8)$ momentary affect reports.

\section{Analytical Approach}


Measuring Affect Dynamics Accurately. To analyze the number of reports required per individual to estimate each of the seven core affect dynamics measures reliably, we began by estimating their "true" value using the complete set of observations available for each individual. For example, if a participant provided 150 momentary affect reports, we computed the seven core affect dynamics measures for this participant (e.g., average happiness, within-person standard deviation, autoregressive coefficient) using all 150 observations. Then, we randomly selected a subset of $N$ affect reports for each individual (with $N$ varying from 3 to 30 ) and computed the affect dynamics measures using this smaller set of observations. We repeated this process 1000 times for each participant and for each value of $N$. We calculated an individual's root mean square error (RMSE) of the estimates (compared to the "true" measure based on the full sample) for each value of $N$. We averaged the RMSE across participants to examine how the accuracy of the estimates changes as one increases the number of reports used to compute the different affect dynamics measures. To provide intuitive benchmarks against which these RMSE values can be compared, we also report, for each affect dynamics measure, the standard deviation of the "true" value in our population. This allows readers to appraise how big or small an RMSE is. For instance, if we were measuring people's weight, an RMSE of 1 gram would be considered very small because the standard deviation of weights in the population is several kilograms. But if we were measuring insects' weights, an RMSE of 1 gram would be considerably larger. If for a given affect dynamics measure and number of affect reports per individual, our average RMSE equals one standard deviation in the true affect dynamics measure across individuals, we can expect the within-person estimation error to be equal in size to one between-person standard deviation in the true measure.

Optimizing Sampling Approaches. Could researchers reduce estimation errors of affect dynamics measures — and thus the number of reports required per individual— by 
probing participants at specific moments? To test whether sampling strategies can be optimized, we compared the accuracy of affect dynamics measures computed using reports selected at random times with affect dynamics measures computed (1) with temporally close or distant reports, (2) reports obtained at specific times, and (3) reports obtained on specific days (see details below).

To assess the accuracy of affect dynamics measures calculated using reports elicited at random times, we followed the same procedure as before: For a given individual and number of reports between 3 and 30, we (1) resampled without replacement and estimated the affect dynamics measures 1000 times, (2) we estimated an individual's root mean square error (RMSE) for each number of reports based on the full sample "true" estimates of the affect dynamics measures, and (3) we averaged the RMSE across individuals for each number of reports used in the computation of the affect dynamics measures. These baseline accuracy estimates are then compared to those obtained using alternative sampling strategies. To assess our results' robustness, for each condition and number of reports used, we bootstrapped over the individual-specific RMSE estimates to obtain the $95 \%$ confidence intervals for the average RMSE across individuals.

Random, close or distant sampling. Close sampling consists in collecting many reports over a short period of time. In this study, we consider close sampling to be the set of consecutive affect reports that were collected within the shortest possible time period for each individual (imposing a maximum of 24 hours between each affect report). In contrast, distant sampling consists in collecting reports less frequently but for a longer period of time. In this study, we consider distant sampling to be the individual's maximally distant reports. To determine an individual's maximally distant reports, we divided the temporal window in which each participant provided reports (from their first to their last) into $N$ - 1 equally spaced time intervals (where $N$ takes on values between 3 and 30, depending on the number 
of reports used in the computation). We then computed the different affect dynamics measures selecting reports that fell as close as possible to an equally spaced design. Note that by construction there is only a single set of reports for each individual that is considered close and distant sampling. Thus, for these sampling strategies, only one value of each affect dynamics measure was calculated per individual for each value of $N$ (instead of resampling and estimating them 1000 times).

Random vs. specific times sampling. Specific times sampling differs from random sampling in that we estimated the affect dynamics measures using reports collected exclusively in the morning (from 6 am to $12 \mathrm{pm}$ ), afternoon (12 pm to $4 \mathrm{pm}$ ), evening (4 pm to $8 \mathrm{pm}$ ), or at night ( $8 \mathrm{pm}$ to $6 \mathrm{am}$ ). For each of these conditions and number of affect reports from 3 to 30, we resampled and estimated the affect dynamics measures 1000 times. We introduced a bias-correction term in the estimates of affect dynamics measures to account for any baseline differences that might exist between specific sampling times (e.g., on average, affect tends to be more pleasant in the evening than in the morning). Results obtained when excluding this bias-correction term can be found in Supplementary Note 2. For each time window, we excluded from our estimations participants that had not provided a minimum of 30 affect reports within that time window.

Random vs. specific days sampling. Specific days sampling differs from random sampling in that we estimated the affect dynamics measures using reports collected exclusively during the weekends (weekend sampling) or during the week (weekday sampling). For each of these conditions, we resampled and estimated each affect dynamics measure 1000 times using a specific number of reports from 3 to 30 . Again, we included a bias-correction procedure and omitted the data from participants that did not provide a minimum of 30 affect reports in each condition. 
Statistical Power as a Function of Sampling. We first estimated the seven affect dynamics measures for each individual using all the observations at our disposal. We then simulated random variables displaying a weak (Pearson's $r=.10$ ), medium $(r=.30)$, and strong $(r=.50)$ positive correlation with each affect dynamics measure by adding orthogonal random Gaussian noise to projections of our variables of interest on vectors displaying the desired correlations. In doing so, we obtained variables displaying a weak, medium, and strong correlation with the affect dynamics measures derived from our full sample. To evaluate how the power to detect these correlations changes when affect dynamics measures are computed from smaller numbers of participants and smaller number of observations per participant, we considered ten different numbers of participants $\left(N_{\text {Participants }}=10,20,40,80\right.$, $160,320,640,1280,2560$, and 5120) and ten different numbers of observations per participants $\left(N_{\text {Observations }}=5,10,15,20,25,30,35,40,45\right.$, and 50$)$, leading to $100(=10 \times 10)$ sampling specifications in total.

For each combination of number of participants and number of observations per participant, we created 2,500 datasets by resampling from our original data. For each of these 2,500 datasets, we computed the seven affect dynamics measures for each participant. We then analyze the correlation between these measures and the simulated variables. We quantified power as the proportion of simulated datasets with a statistically significant positive correlation between the affect dynamics measures and the simulated variable.

Benchmarks for Plausible Effect Sizes. Like other power calculation tools, the sampling recommendations derived from our empirical framework require researchers to anticipate plausible effect sizes for the association they are interested in (or to set a minimum effect size that they want their study to detect). Such anticipated effect sizes can be informed by systematic literature review, preliminary data, and meta-analyses. But in practice, it may be challenging for affective scientists to come up with realistic effect size estimates as the 
field of affect dynamics is relatively new, and such estimates may not exist. Moreover, historical data may offer little guidance as past estimates tend to be overestimates given reporting and publication bias favoring significant results (Gelman \& Carlin, 2014). Therefore, we provide a series of benchmarks based on 10 variables that we measured alongside affect in our experience-sampling project: (1) age, (2) gender, (3) average sleep time, (4) life satisfaction, (5) meaning in life, as well as the proportion of time spent with (6) friends, (7) family, (8) alone, (9) working, and (10) exercising (see Supplementary Note 5 for the complete list of variables and their operationalizations). Note that for life satisfaction and meaning in life, the associations we report are based on matched measures. For instance, we report the correlation between trait affect and trait life satisfaction, the correlation between affect instability and life satisfaction instability, and the correlation between affect inertia and life satisfaction inertia (vs. non-matching pairs).

By considering the magnitude of the relationships between these ten variables and the different affect dynamics measures, we hope to help researchers design optimized ESM studies based on plausible effect size estimates.

\section{Results}

\section{Measuring Affect Dynamics Accurately}

Figure 2 depicts changes in RMSE as we increase the number of observations per individual used to compute the seven affect dynamics measures. Our results show a large degree of heterogeneity between measures. We found that the number of observations needed to estimate our affect dynamics measures with a minimum accuracy of one between-subject standard deviation in the true measures ranges from 3 for trait affect to over 30 for the autocorrelation coefficient. 

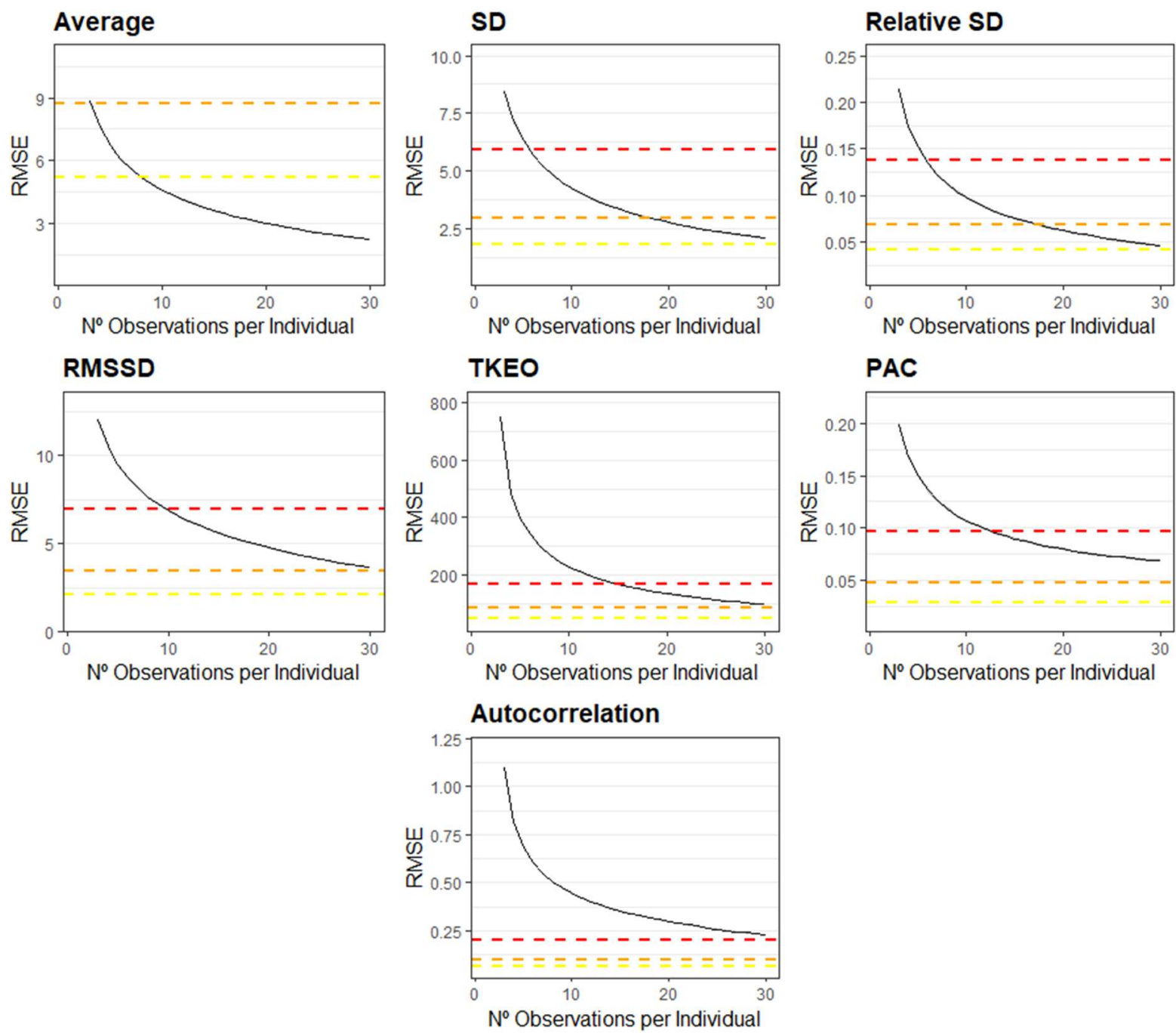

Figure 2. Average RMSE in the estimation of affect dynamics measures as a function of the number of observations per participant. Dashed lines provide accuracy benchmarks depicting 1 (red), 0.5 (orange), and 0.3 (yellow) between-subjects standard deviations in the affect dynamics measure estimated on the full sample.

\section{Optimizing Sampling Approaches}

Random, close or distant sampling. Is it better to conduct short intense studies or longer less-demanding ones? As shown in Figure 3, the optimal measurement method depends on the affect dynamics measure of interest and the number of observations used to estimate it. We found large differences in the estimation error across sampling methods when calculating affect dynamics measures that are not temporally dependent (i.e., average affect, 
standard deviation, and relative standard deviation). Estimations of these three measures under close sampling were significantly less accurate than under random and distant sampling. For example, we can estimate a person's average affect more accurately with ten observations collected at random times over multiple days or weeks than with over 30 consecutive observations over shorter periods of time. In addition, when only a few observations can be collected, we found that distant sampling leads to more accurate estimations than both close and random sampling. Note that the difference between distant and random sampling is small and not statistically significant when at least 27 observations per individual are included in the estimation.

The differences in accuracy across sampling methods were substantially smaller and less consistent for temporally dependent affect dynamics measures (i.e., RMSSD, autocorrelation coefficient, TKEO, and PAC). For RMSSD and the autocorrelation coefficient, estimates obtained through close and distant sampling did not differ, though both of these strategies outperformed random sampling. For TKEO, close sampling largely outperformed both distant and random sampling, especially when the number of observations per participant is small. For the PAC, distant sampling outperformed close and random sampling, especially when the number of observations per participant is large. 

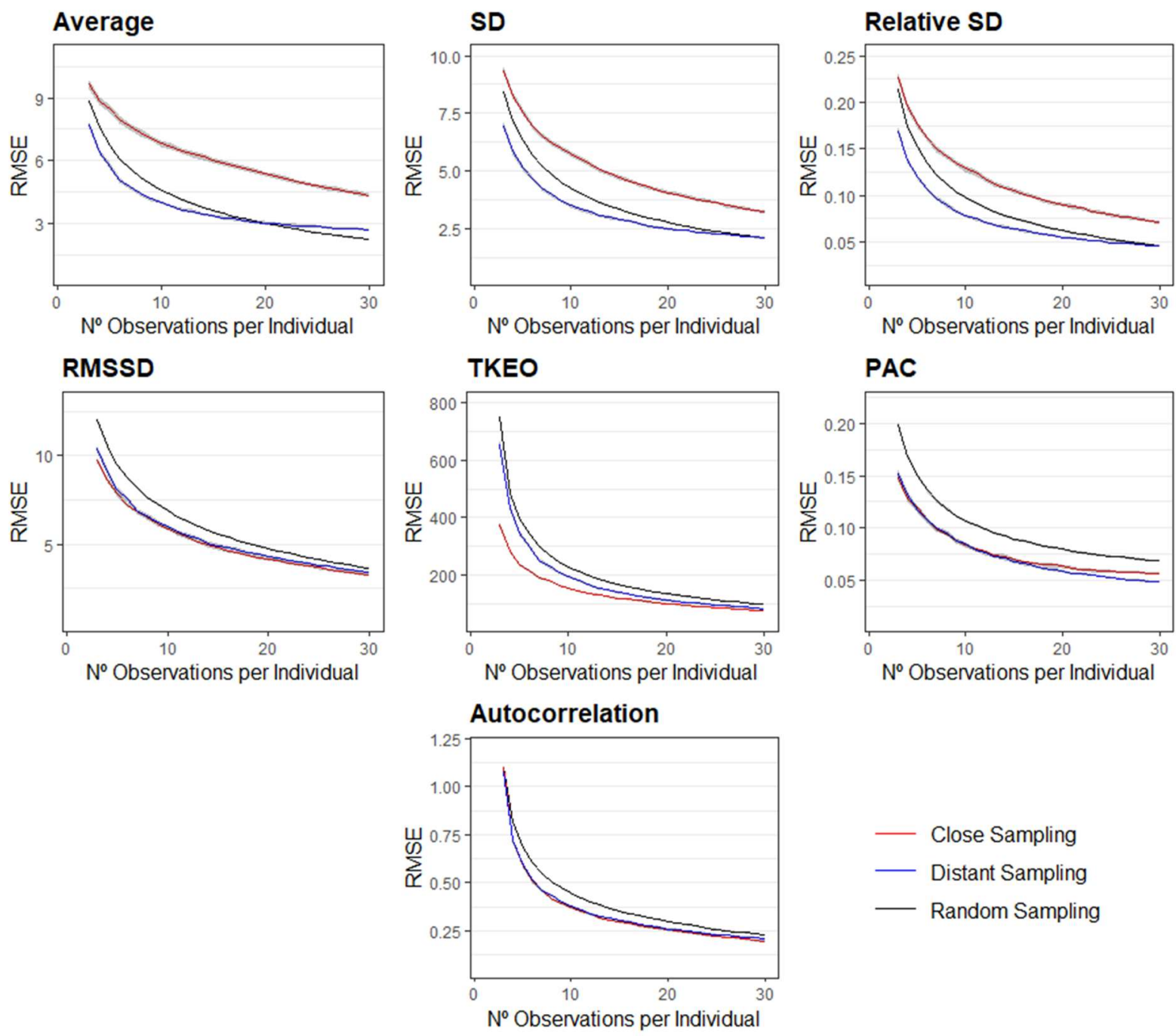

Figure 3. Average RMSE in the estimation of affect dynamics measures as a function of the number of observations per participant collected under random (black), close (red), or distant (blue) sampling. Gray areas around the lines represent the $95 \%$ confidence intervals for the average RMSE.

Random vs. specific times sampling. Are there better moments than others to capture people's affective states? For non-temporally dependent measures (i.e., average affect, standard deviation, and relative standard deviation), random sampling tended to outperform estimates based solely on observations collected at specific times-with estimates based on night hours leading to the highest estimation error (see Supplementary Figure S1S4). Note that the differences were small and, in many cases, non-significant. For affect 
instability measures (i.e., RMSSD, TKEO, PAC) sampling exclusively at specific times outperforms random sampling, although the differences are small and non-significant across most numbers of samples. Sampling earlier in the day, either in the morning or in the afternoon yielded the best results. For affect inertia (i.e., autocorrelation coefficient), sampling exclusively at specific times performed better than random sampling — with estimates based on night hours providing the best performance. Detailed results for random vs. specific times sampling can be found in Supplementary Note 2.

Random vs. specific days sampling. Are there better days than others to capture people's affect dynamics? For non-temporally dependent measures (i.e., average affect, standard deviation, and relative standard deviation), random sampling tended to perform better than sampling on specific days - with estimates based on weekend observations yielding the highest estimation error. Again, these differences were small and, in many cases, non-significant. For measures of affect instability, we did not find differences between random sampling and sampling on specific days for TKEO and PAC, but we found small differences favoring sampling on the weekends for the estimation of the RMSSD. For affect inertia (i.e., autocorrelation coefficient), sampling exclusively on the weekends and sampling exclusively on the weekdays performed better than random sampling-with sampling on the weekends yielding the best performance. Detailed results for random vs. specific days sampling can be found in Supplementary Note 2.

\section{Statistical Power as a Function of Sampling}

Figure 4 displays the minimal combinations of number of individuals and observations per individuals needed to achieve $80 \%$ power to detect an association of medium size $(r=.30)$ using an alpha of .05. The different curves are intended to provide a quick overview of how the number of individuals and samples per individuals can be traded off. Detailed information about (1) the method we used to estimate these curves, (2) the specific power achieved for all 
tested combinations of number of individuals and samples per individual, and (3) other effect sizes and power levels are presented in Supplementary Note 3 and in the online app (https://sergiopirla.shinyapps.io/powerADapp).

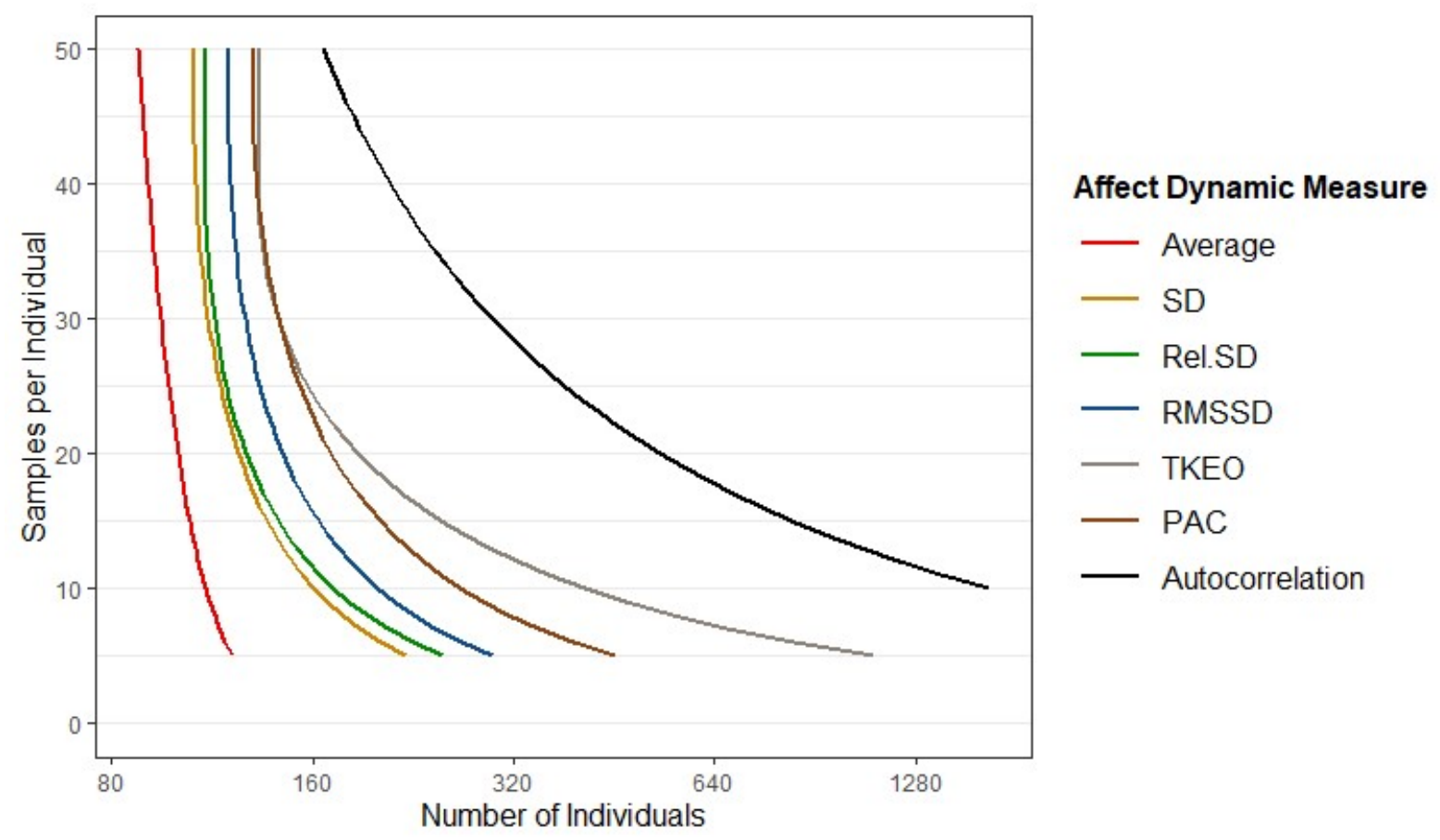

Figure 4. Minimum number of individuals and samples per individual required to achieve sufficient power $(\geq 80 \%)$ to detect a correlation of medium effect size $(r=.30)$ with an alpha of 0.05 . The $\mathrm{x}$-axis is in log-scale.

Adequate power could be achieved with a relatively small number of observations per individual. As a general rule, as long as a study includes at least 200 participants, sampling 20 observations per individual yields sufficient power for most affect dynamics measures. For average affect, standard deviation, and relative standard deviation, sufficient power was even achieved with 5 to 10 observations for 200 individuals. For measures of affect instability (i.e., RMSSD, PAC, and TKEO), 20 observations for 200 individuals were required. The only exception to the $200 \times 20$ rule arises with affect inertia (i.e., 
autocorrelation coefficient), for which over 40 observations for 200 individuals were required. It is important to note that these sample recommendations apply to studies with an expected medium-size association of interest $(r=.30)$. However, as our plausible effect sizes benchmarks suggest, many affect dynamics measures display relatively weak associations with demographic, well-being, and time-allocation outcomes (see next section).

Overall, averaging across the range of all sampling combinations, affect dynamics measures, alpha levels, and effect sizes, increasing the number of individuals had a larger impact on power than increasing the number of observations per individual-with the exception of affect inertia which showed the opposite pattern (see Note 4 of Supplementary Materials).

\section{Benchmarks for Plausible Effect Sizes}

In power calculation, researchers are asked to anticipate the effect sizes of their associations of interest or to decide on a minimum effect size that they are willing to detect. How can one know in advance what plausible effect sizes might be? Figure 5 displays the magnitude of the associations between affect dynamics measures and ten outcomes: (1) age, (2) gender, (3) average sleep time, (4) life satisfaction, (5) meaning in life, as well as the proportion of time spent with (6) friends, (7) family, (8) alone, (9) working, and (10) exercising. As noted in the methods section, for life satisfaction and meaning, we estimated the association between dynamic measures of these variables and their corresponding affect dynamics measures. These values can be used as broad benchmarks when attempting to postulate plausible effect sizes (see Supplementary Note 5 for additional information and results). For example, researchers interested in examining the relationship between average affect and the propensity to eat carrots could ask themselves whether they expect this relationship to be smaller or greater than the link between average affect and age $(r=.06)$, time spent alone $(r=-.24)$, or trait meaning in life $(r=.84)$. Likewise, researchers interested 
in examining the relationship between affect instability and family history of bipolar disorder could ask themselves whether they expect the relationship to be smaller or greater than the link between affect instability and time spent with friends $(r=.10)$, age $(r=-.27)$, or life satisfaction instability $(r=.34)$.
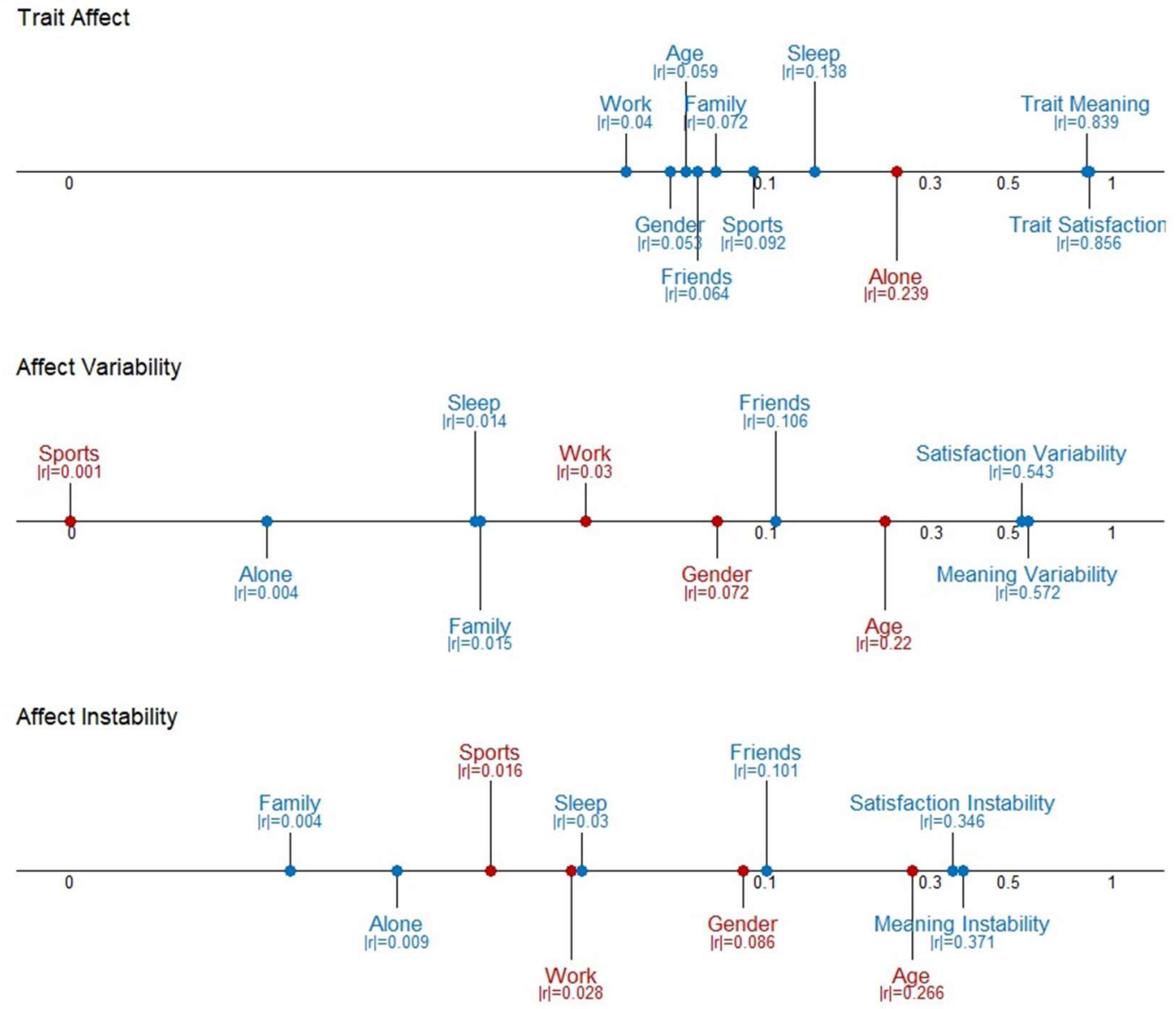

Affect Inertia

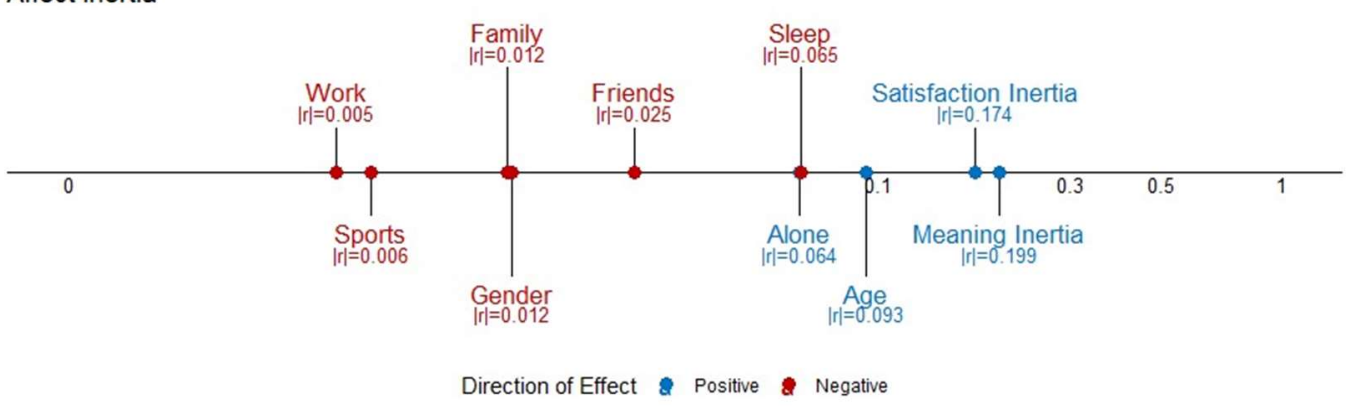

Figure 5. Correlations between affect dynamics measures and different outcome variables in our dataset. Positive and negative correlations are presented in blue and red, respectively. 
Building on our results and expanding our power calculations to all effect sizes, we developed an R-package (“powerAD”) and a Shiny App (https://sergiopirla.shinyapps.io/powerADapp/) to help researchers make empiricallyinformed decisions about study design of affect dynamics studies. We refer to the package site (https://sergiopirla.github.io/powerAD) for more information on how to download, install and run its primary functions.

Our Shiny app is composed of two main panels. On the first panel ("Sampling Calculator"), users can estimate a set of valid sampling approaches for each affect dynamics measure given a specified statistical power, effect size, and alpha level. On the second panel ("Power Calculator"), users can estimate the statistical power achieved by a specific study based on its characteristics (sampling approach, affect dynamics measure, effect size, and alpha level). For example, panel A of Figure 6 shows the minimal combinations of number of individuals and number of observations per individual to obtain a statistical power of $80 \%$ to detect an $r=.30$ at the 5\% significance level for the Taeger Kaiser Energy Operator (TKEO). Panel B provides the precise power estimate for the same $r=.30$ effect size and TKEO measure given a specific sample of 400 participants each surveyed 11 times. Finally, the app also provides a series of benchmark effect sizes for each affect dynamics measures to help researchers estimate plausible effect sizes. 


\section{Panel A:}

\section{Statistical Power Calculator for Affect Dynamics Studies}

Sample Size Calculator Power Calculator Effect Sizes

Sample Size Calculator: Estimates the combinations of participants and reports per participants needed to achieve a minimum specified power given an affect dynamic measure of interest, an effect size, and an alpha level. Based on Pirla, Taquet and Quoidbach (2021).

\begin{tabular}{|c|c|c|c|c|}
\hline \multicolumn{2}{|l|}{ Power } & \multicolumn{3}{|c|}{$\begin{array}{l}\text { Number of individuals and affect reports per individual (samples) needed to achieve a power } \\
\text { of } 0.8 \text { or more to detect an effect of size } r=0.3 \text { using an alpha of } 0.05 \text {. }\end{array}$} \\
\hline 0,8 & $\hat{\imath}$ & Power ${ }^{*}$ & Individuals & Samples \\
\hline \multicolumn{2}{|c|}{ Affect Dynamic Measure } & 0.80 & 1200 & 5 \\
\hline TKEO & $\vec{r}$ & 0.81 & 360 & 10 \\
\hline \multicolumn{2}{|c|}{ Effect Size (as Pearson's r) } & 0.80 & 240 & 15 \\
\hline \multirow{2}{*}{0,3} & \multirow{2}{*}{$\hat{\imath}$} & 0.80 & 180 & 20 \\
\hline & & 0.81 & 170 & 25 \\
\hline \multicolumn{2}{|l|}{ Alpha Level } & 0.83 & 150 & 30 \\
\hline \multirow[t]{2}{*}{0.05} & 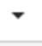 & 0.81 & 140 & 35 \\
\hline & & 0.81 & 130 & 45 \\
\hline \multirow{7}{*}{ Calculate } & & & & \\
\hline & & \multicolumn{3}{|c|}{$\begin{array}{l}\text { *Power is estimated through a linear interpolation using the sample combinations included } \\
\text { in our main analyses. We refrain from making power extrapolations and therefore, only } \\
\text { consider sampling approaches that range between } 10 \text { and } 5120 \text { participants and from } 5 \text { to } \\
50 \text { affect reports per participant (samples). The following table presents the minimal } \\
\text { sampling combinations included in our main analyses that yielded the specified power: }\end{array}$} \\
\hline & & Power & Individuals & Samples \\
\hline & & 0.85 & 1280 & 5 \\
\hline & & 0.97 & 640 & 10 \\
\hline & & 0.93 & 320 & 15 \\
\hline & & 0.87 & 160 & 30 \\
\hline
\end{tabular}




\section{Panel B:}

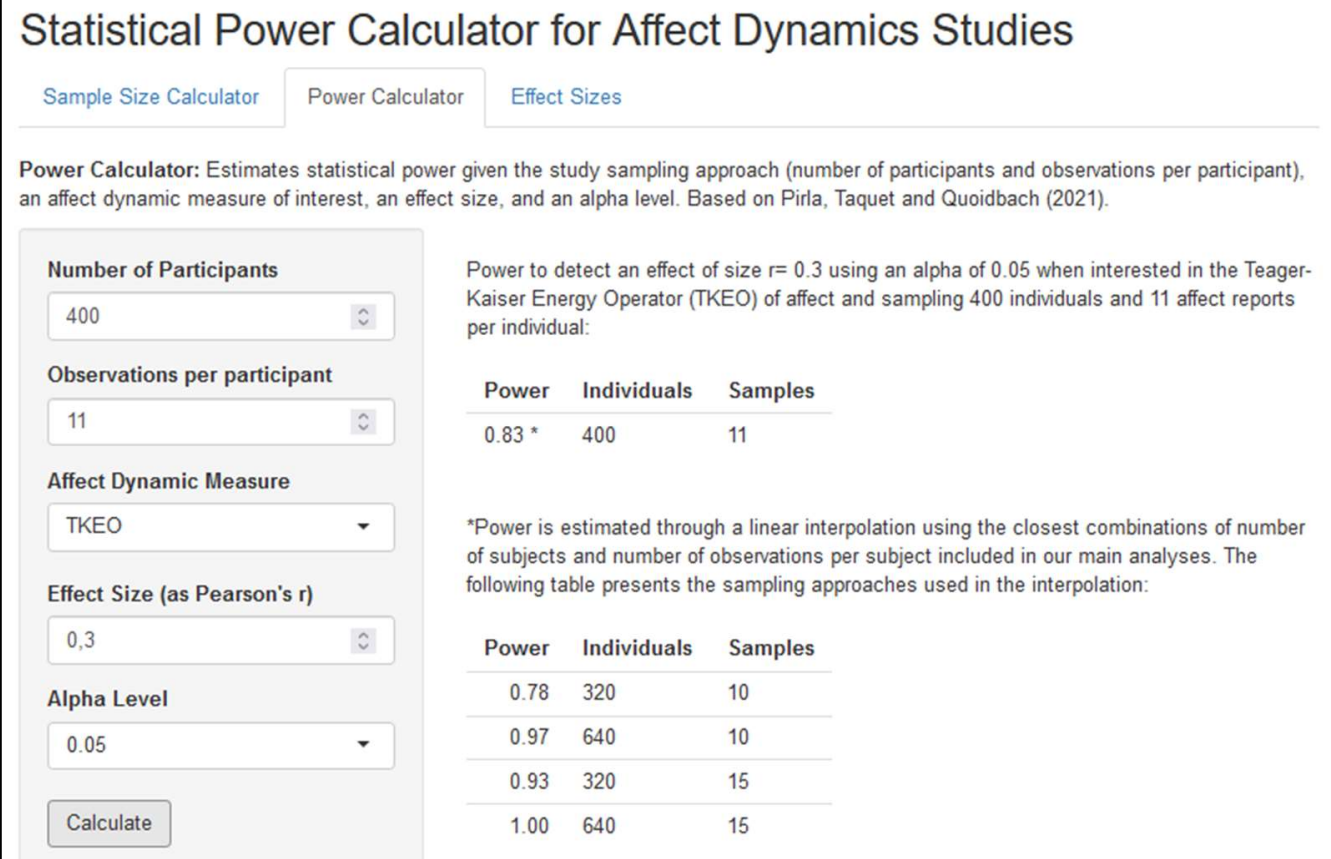

Figure 6. Shiny App to calculate power in affect dynamics studies. Panel A shows the sample size calculator. Panel B shows the power calculator.

\section{Discussion}

This paper introduces an empirically-derived framework to help researchers design well-powered and efficient experience-sampling studies in the growing field of affect dynamics. Whereas the ideal sampling approach depends on the specific affect dynamics measure under consideration, three design principles emerge from our research. First, a sample of 200 participants each providing 20 observations (i.e., $200 \times 20$ rule) yields sufficient power to detect medium size associations for most affect dynamics measures. Second, the optimal sampling strategy depends on the affect dynamics measure of interest. For trait affect and affect variability, it is often better to run longer less-demanding studies (i.e., few daily measurements spread out over several weeks) than shorter intense ones (i.e., many daily affect measurements spread out over several days). For measures of instability 
and inertia, both short intense studies and longer less-demanding studies outperform random samples with little difference between the two designs. Third, little differences were observed between random sampling and sampling at specific times or on specific days, so that the choice of sampling moments can be dictated by other considerations (such as the individual's preferences or practicalities related to the study at hand).

The novel framework and tools developed here further suggest that the literature on affect dynamics is largely underpowered. From the 88 affect dynamics studies we reviewed in the Introduction, $45 \%$ of the analyses fail to reach an $80 \%$ power to detect a medium correlation $(r=.30)$ with a $5 \%$ significance level. And this figure could be even more concerning given that many of the relationships between affect dynamics and outcomes we provide as benchmarks (e.g., demographics, lifestyle) show smaller effect sizes (consistent with typical effect sizes reported in affective and behavioral sciences; Bosco et al., 2015; Richard \& Bond, 2003). When we consider the power to detect a small effect $(r=.10)$ with a $5 \%$ significance level, we find that the average statistical power achieved with the sample sizes of these 88 studies ranges between $10 \%$ and $16 \%$ depending on the affect dynamics measure of interest (see Note 6 of Supplementary Material). The relatively low power and measurement error issues associated with complex affect dynamics measures like instability and inertia could explain, in part, recent findings by Dejonckheere and colleagues (2019) suggesting that these measures have little predictive value over the mean and variance.

The present study provides a robust empirical framework to conduct experiencesampling studies in affective science. But it is important for future research to address several limitations. First, our "true" values (i.e., those based on all the available measurements for an individual) were based on at least 50 observations per participant. However, it might be that more extensive data at the participant level (e.g., 1000 observations per individual) would lead to somewhat different inferences. Second, our recommendation about when researchers 
should survey participants is limited to relatively basic strategies (e.g., random moments vs. specific days or times). Future research is needed to examine whether advanced contextaware strategies (e.g., sending surveys in response to changes in participants' environmental or psychological circumstances) lead to substantial gains in accuracy and statistical power. Third, although we relied on an exceptionally large sample, our participants may not be representative of the general population. Future research is also needed to examine whether our recommendations need to be adjusted for specific groups of people (e.g., patients with depression, older adults). Fourth, our recommendations are based on accuracy and statistical power considerations. They do not take into account how different sampling strategies may affect burden, compliance, and careless responding in experience-sampling research. While recent research suggests that sampling frequency has no impact on participant's burden, data quantity, and data quality (Eisele et al., 2020), further research is needed to examine whether other recommendations derived from our findings are similarly free of negative consequences. Finally, our framework focused on a general, unidimensional measure of affect (unhappy - happy) and the optimal sampling strategies to detect correlations. In future research, it is important to examine how our recommendations apply to other affective states, including specific emotions, and non-linear relationships between affect dynamics measures and outcomes. We hope that the data and code provided will allow researchers to expand our framework, opening the door to fast and exciting advances in the study of human emotions.

\section{Open Practices Statement:}

Data and code to replicate the main analyses presented in this paper will be made available upon publication in an OSF repository. Interested readers are also referred to our Shinny App (https://sergiopirla.shinyapps.io/powerADapp/) and R-package

(https://sergiopirla.github.io/powerAD/). 


\section{References}

Aan het Rot, M., Hogenelst, K., \& Schoevers, R. A. (2012). Mood disorders in everyday life: A systematic review of experience sampling and ecological momentary assessment studies. Clinical Psychology Review, 32, 510-523.

Aguinis, H., Gottfredson, R. K., \& Culpepper, S. A. (2013). Best-Practice

Recommendations for Estimating Cross-Level Interaction Effects Using Multilevel Modeling. Journal of Management, 39, 1490-1528.

Anestis, M. D., Selby, E. A., Crosby, R. D., Wonderlich, S. A., Engel, S. G., \& Joiner, T. E. (2010). A comparison of retrospective self-report versus ecological momentary assessment measures of affective lability in the examination of its relationship with bulimic symptomatology. Behaviour Research and Therapy, 48, 607-613.

Arend, M. G., \& Schäfer, T. (2019). Statistical power in two-level models: A tutorial based on Monte Carlo simulation. Psychological Methods, 24, 1-19.

Arslan, R. C., Walther, M. P., \& Tata, C. S. (2019). formr: A study framework allowing for automated feedback generation and complex longitudinal experience-sampling studies using R. Behavior Research Methods, 52, 376-387.

Astivia, O. L., Gadermann, A., \& Guhn, M. (2019). The relationship between statistical power and predictor distribution in multilevel logistic regression: a simulation-based approach. BMC Medical Research Methodology, 19, 97-117.

Beal, D. J., \& Ghandour, L. (2010). Stability, change, and the stability of change in daily workplace affect. Journal of Organizational Behavior, 32, 526-546.

Bosco, F. A., Aguinis, H., Singh, K., Field, J. G., \& Pierce, C. A. (2015). Correlational effect size benchmarks. The Journal of applied psychology, 100, 431-449.

Bowen, R., Baetz, M., Hawkes, J., \& Bowen, A. (2006). Mood variability in anxiety disorders. Journal of Affective Disorders, 91, 165-170. 
Bowen, R., Clark, M., \& Baetz, M. (2004). Mood swings in patients with anxiety disorders compared with normal controls. Journal of Affective Disorders, 78, 185-192.

Calamia, M. (2019). Practical considerations for evaluating reliability in ambulatory assessment studies. Psychological Assessment, 31, 285-291.

Chepenik, L. G., Have, T. T., Oslin, D., Datto, C., Zubritsky, C., \& Katz, I. R. (2006). A Daily Diary Study of Late-Life Depression. The American Journal of Geriatric Psychiatry, 14, 270-279.

Choi, J., Catapano, R., \& Choi, I. (2016). Taking Stock of Happiness and Meaning in Everyday Life. Social Psychological and Personality Science, 8, 641-651.

Conner, T. S., Tennen, H., Fleeson, W., \& Barrett, L. F. (2009). Experience Sampling Methods: A Modern Idiographic Approach to Personality Research. Social and Personality Psychology Compass, 3, 292-313

Csikszentmihalyi, M., \& Larson, R. (1984) Being adolescent: Conflict and growth in the teenage years. New York: Basic Books.

Deaton, A., \& Stone, A. A. (2016). Understanding context effects for a measure of life evaluation: how responses matter. Oxford Economic Papers, 68, 861-870.

Delespaul, P. A., \& deVries, M. W. (1987). The Daily Life of Ambulatory Chronic Mental Patients. The Journal of Nervous and Mental Disease, 175, 537-544.

Dejonckheere, E., Mestdagh, M., Houben, M., Erbas, Y., Pe, M., Koval, P., . . Kuppens, P. (2018). The bipolarity of affect and depressive symptoms. Journal of Personality and Social Psychology, 114, 323-341.

Dejonckheere, E., Mestdagh, M., Houben, M., Rutten, I., Sels, L., Kuppens, P., \& Tuerlinckx, F. (2019). Complex affect dynamics add limited information to the prediction of psychological well-being. Nature Human Behaviour, 3, 478-491. 
Dunster, G. P., Swendsen, J., \& Merikangas, K. R. (2020). Real-time mobile monitoring of bipolar disorder: a review of evidence and future directions.

Neuropsychopharmacology, 46, 197-208.

Ebner-Priemer, U. W., Kuo, J., Kleindienst, N., Welch, S. S., Reisch, T., Reinhard, I., Lieb, K., Linehan, M. M., \& Bohus, M. (2007). State affective instability in borderline personality disorder assessed by ambulatory monitoring. Psychological Medicine, 37, 961-970.

Ebner-Priemer, U. W., \& Trull, T. J. (2009). Ambulatory Assessment. European Psychologist, 14, 109-119.

Eisele, G., Vachon, H., Lafit, G., Kuppens, P., Houben, M., Myin-Germeys, I., \& Viechtbauer, W. (2020). The effects of sampling frequency and questionnaire length on perceived burden, compliance, and careless responding in experience sampling data in a student population. Assessment, 1073191120957102.

Ellison, W. D., Trahan, A. C., Pinzon, J. C., Gillespie, M. E., Simmons, L. M., \& King, K. Y. (2020). For whom, and for what, is experience sampling more accurate than retrospective report? Personality and Individual Differences, 163, 110071.

Fisher, C. D., \& To, M. L. (2012). Using experience sampling methodology in organizational behavior. Journal of Organizational Behavior, 33, 865-877.

Fredrickson, B. L., \& Kahneman, D. (1993). Duration neglect in retrospective evaluations of affective episodes. Journal of Personality and Social Psychology, 65, 45-55.

Gelman, A., \& Carlin, J. (2014). Beyond Power Calculations: Assessing type S (sign) and type M (magnitude) errors. Perspectives on Psychological Science, 9, 641-651.

Golier, J. A., Yehuda, R., Schmeidler, J., \& Siever, L. J. (2001). Variability and severity of depression and anxiety in post traumatic stress disorder and major depressive disorder. Depression and Anxiety, 13, 97-100. 
Gruber, J., Kogan, A., Quoidbach, J., \& Mauss, I. B. (2013). Happiness is best kept stable: Positive emotion variability is associated with poorer psychological health. Emotion, $13,1-6$.

Havermans, R., Nicolson, N. A., \& deVries, M. W. (2007). Daily Hassles, Uplifts, and Time Use in Individuals With Bipolar Disorder in Remission. Journal of Nervous \& Mental Disease, 195, 745-751.

Houben, M., Van Den Noortgate, W., \& Kuppens, P. (2015). The relation between shortterm emotion dynamics and psychological well-being: A meta-analysis. Psychological Bulletin, 141, 901-930.

Ioannidis, J. P. A. (2005). Why Most Published Research Findings Are False. PLoS Medicine, 2, e124.

Ioannidis, J. P. A. (2008). Why Most Discovered True Associations Are Inflated. Epidemiology, 19, 640-648.

Jahng, S., Wood, P. K., \& Trull, T. J. (2008). Analysis of affective instability in ecological momentary assessment: Indices using successive difference and group comparison via multilevel modeling. Psychological Methods, 13, 354-375.

Jones, S. H., Hare, D. J., \& Evershed, K. (2005). Actigraphic assessment of circadian activity and sleep patterns in bipolar disorder. Bipolar Disorders, 7, 176-186.

Kahneman, D., Fredrickson, B. L., Schreiber, C. A., \& Redelmeier, D. A. (1993). When More Pain Is Preferred to Less: Adding a Better End. Psychological Science, 4, 401405.

Kerr, M. L., Rasmussen, H. F., Buttitta, K. V., Smiley, P. A., \& Borelli, J. L. (2020). Exploring the complexity of mothers' real-time emotions while caregiving. Emotion, 21, $545-556$. 
Kirtley, O. J., Lafit, G., Achterhof, R., Hiekkaranta, A. P., \& Myin-Germeys, I. (2021). Making the black box transparent: A template and tutorial for registration of studies using experience-sampling methods. Advances in Methods and Practices in Psychological Science, 4, 251524592092468.

Knowles, R., Tai, S., Jones, S. H., Highfield, J., Morriss, R., \& Bentall, R. P. (2007). Stability of self-esteem in bipolar disorder: Comparisons among remitted bipolar patients, remitted unipolar patients and healthy controls. Bipolar Disorders, 9, 490-495.

Kuppens, P. (2015). It's about time: A special section on affect dynamics. Emotion Review, 7, 297-300.

Kuppens, P., Allen, N. B., \& Sheeber, L. B. (2010). Emotional inertia and psychological maladjustment. Psychological Science, 21, 984-991.

Kuppens, P., \& Verduyn, P. (2017). Emotion dynamics. Current Opinion in Psychology, 17, $22-26$.

Lane, S. P., \& Hennes, E. P. (2018). Power struggles: Estimating sample size for multilevel relationships research. Journal of Social and Personal Relationships, 35, 7-31.

Lapate, R. C., \& Heller, A. S. (2020). Context matters for affective chronometry. Nature Human Behaviour, 4, 688-689.

Links, P. S., Heisel, M. J., \& Garland, A. (2003). Affective instability in personality disorders. American Journal of Psychiatry, 160, 394-395.

Lucas, R. E., Wallsworth, C., Anusic, I., \& Donnellan, M. B. (2021). A direct comparison of the day reconstruction method (DRM) and the experience sampling method (ESM). Journal of Personality and Social Psychology, 120, 816-835.

McConville, C., \& Cooper, C. (1996). Mood variability and the intensity of depressive states. Current Psychology, 14, 329-338. 
Meers, K., Dejonckheere, E., Kalokerinos, E. K., Rummens, K., \& Kuppens, P. (2020). mobileQ: A free user-friendly application for collecting experience sampling data. Behavior Research Methods, 52, 1510-1515.

Mestdagh, M., Pe, M., Pestman, W., Verdonck, S., Kuppens, P., \& Tuerlinckx, F. (2018). Sidelining the mean: The relative variability index as a generic mean-corrected variability measure for bounded variables. Psychological Methods, 23, 690-707.

Mueller, S., Ram, N., Conroy, D. E., Pincus, A. L., Gerstorf, D., \& Wagner, J. (2019). Happy like a fish in water? The role of personality-situation fit for momentary happiness in social interactions across the adult lifespan. European Journal of Personality, 33, 298-316.

Myin-Germeys, I., Delespaul, P. A., \& deVries, M. W. (2000). Schizophrenia patients are more emotionally active than is assumed based on their behavior. Schizophrenia Bulletin, 26, 847-854.

Myin-Germeys, I., Kasanova, Z., Vaessen, T., Vachon, H., Kirtley, O., Viechtbauer, W., \& Reininghaus, U. (2018). Experience sampling methodology in mental health research: new insights and technical developments. World Psychiatry, 17, 123-132.

Myin-Germeys, I., Oorschot, M., Collip, D., Lataster, J., Delespaul, P., \& van Os, J. (2009). Experience sampling research in psychopathology: Opening the black box of daily life. Psychological Medicine, 39, 1533-1547.

Nesselroade, J. R., \& Salthouse, T. A. (2004). Methodological and theoretical implications of intraindividual variability in perceptual-motor performance. The Journals of Gerontology Series B: Psychological Sciences and Social Sciences, 59, 49-55.

Peeters, F., Berkhof, J., Delespaul, P., Rottenberg, J., \& Nicolson, N. A. (2006). Diurnal mood variation in major depressive disorder. Emotion, 6, 383-391. 
Peeters, F., Berkhof, J., Rottenberg, J., \& Nicolson, N. A. (2010). Ambulatory emotional reactivity to negative daily life events predicts remission from major depressive disorder. Behaviour Research and Therapy, 48, 754-760.

Pfaltz, M. C., Michael, T., Grossman, P., Margraf, J., \& Wilhelm, F. H. (2010). Instability of physical anxiety symptoms in daily life of patients with panic disorder and patients with posttraumatic stress disorder. Journal of Anxiety Disorders, 24, 792-798.

Quoidbach, J., Taquet, M., Desseilles, M., de Montjoye, Y. A., \& Gross, J. J. (2019). Happiness and social behavior. Psychological Science, 30, 1111-1122.

Redelmeier, D. A., \& Kahneman, D. (1996). Patients' memories of painful medical treatments: real-time and retrospective evaluations of two minimally invasive procedures. Pain, 66, 3-8.

Richard, F. D., Bond, C. F., \& Stokes-Zoota, J. J. (2003). One hundred years of social psychology quantitatively described. Review of General Psychology, 7, 331-363.

Santangelo, P., Bohus, M., \& Ebner-Priemer, U. W. (2014). Ecological momentary assessment in borderline personality disorder: A review of recent findings and methodological challenges. Journal of Personality Disorders, 28, 555-576.

Schimmack, U. (2003). Affect measurement in experience sampling research. Journal of Happiness Studies, 4, 79-106.

Schimmack, U., \& Oishi, S. (2005). The influence of chronically and temporarily accessible information on life satisfaction judgments. Journal of Personality and Social Psychology, 89, 395-406.

Scollon, C.N., Kim-Prieto, C., \& Diener, E. (2003). Experience sampling: Promises and pitfalls, strengths and weaknesses. Journal of Happiness Studies, 4, 5-34. 
Solhan, M. B., Trull, T. J., Jahng, S., \& Wood, P. K. (2009). Clinical assessment of affective instability: Comparing EMA indices, questionnaire reports, and retrospective recall. Psychological Assessment, 21, 425-436.

Solnik, S., Rider, P., Steinweg, K., DeVita, P., \& Hortobágyi, T. (2010). Teager-Kaiser energy operator signal conditioning improves EMG onset detection. European Journal of Applied Physiology, 110, 489-498.

Stone, A. A., Schwartz, J. E., Neale, J. M., Shiffman, S., Marco, C. A., Hickcox, M., . . Cruise, L. J. (1998). A comparison of coping assessed by ecological momentary assessment and retrospective recall. Journal of Personality and Social Psychology, 74, $1670-1680$.

Stone, A. A., \& Shiffman, S. (1994). Ecological momentary assessment (EMA) in behavioral medicine. Annals of Behavioral Medicine, 16, 199-202.

Taquet, M., Quoidbach, J., de Montjoye, Y. A., Desseilles, M., \& Gross, J. J. (2016). Hedonism and the choice of everyday activities. Proceedings of the National Academy of Sciences, 113, 9769-9773.

Taquet, M., Quoidbach, J., Gross, J. J., Saunders, K. E. A., \& Goodwin, G. M. (2020). Mood homeostasis, low mood, and history of depression in 2 large population samples. JAMA Psychiatry, 77, 944-951.

Thompson, R. J., Mata, J., Jaeggi, S. M., Buschkuehl, M., Jonides, J., \& Gotlib, I. H. (2012). The everyday emotional experience of adults with major depressive disorder: Examining emotional instability, inertia, and reactivity. Journal of Abnormal Psychology, 121, 819-829.

Trampe, D., Quoidbach, J., \& Taquet, M. (2015). Emotions in Everyday Life. PLOS ONE, $10, \mathrm{e} 0145450$. 
Trull, T. J., \& Ebner-Priemer, U. W. (2020). Ambulatory assessment in psychopathology research: A review of recommended reporting guidelines and current practices. Journal of Abnormal Psychology, 129, 56-63.

Trull, T. J., Lane, S. P., Koval, P., \& Ebner-Priemer, U. W. (2015). Affective Dynamics in Psychopathology. Emotion Review, 7, 355-361.

Trull, T. J., Solhan, M. B., Tragesser, S. L., Jahng, S., Wood, P. K., Piasecki, T. M., \& Watson, D. (2008). Affective instability: Measuring a core feature of borderline personality disorder with ecological momentary assessment. Journal of Abnormal Psychology, 117, 647-661.

Tsanas, A., Saunders, K., Bilderbeck, A., Palmius, N., Osipov, M., Clifford, G., . . . De Vos, M. (2016). Daily longitudinal self-monitoring of mood variability in bipolar disorder and borderline personality disorder. Journal of Affective Disorders, 205, 225-233.

Watson, D., \& Tellegen, A. (1985). Toward a consensual structure of mood. Psychological Bulletin, 98, 219-235.

Wendt, L. P., Wright, A. G. C., Pilkonis, P. A., Woods, W. C., Denissen, J. J. A., Kühnel, A., and Zimmermann, J. (2020). Indicators of affect dynamics: structure, reliability, and personality correlates. European Journal of Personality, 34, 1060-1072.

Zeigler-Hill, V., \& Abraham, J. (2006). Borderline personality features: instability of selfesteem and affect. Journal of Social and Clinical Psychology, 25, 668-687. 
AFFECT DYNAMICS 38

\section{Acknowledgments}

JQ thanks the Ministerio de Economía, Industria y Competitividad, Gobierno de España (RYC-2016-21020) for financial support. MT is an NIHR Academic Clinical fellow and NIHR Oxford Health Biomedical Research Centre Senior Research Fellow (grant BRC1215-20005). The views expressed are those of the authors and not necessarily those of the UK National Health Service, NIHR, or the UK Department of Health. 


\section{Supplementary Materials}

\section{Note 1: Sample Summary Statistics}

The following tables present the summary statistics for the sample of participants included in our analyses:

\begin{tabular}{cccccc}
\hline Sample Variable & \multicolumn{1}{c}{ Average } & SD & Min & \multicolumn{1}{c}{ Max } \\
\hline Age & 29.97 & 9.96 & 13 & 73 \\
$\begin{array}{c}\text { Number of affect } \\
\text { reports per participant }\end{array}$ & 111.55 & 87.79 & 51 & 1689 \\
\hline
\end{tabular}

Supplemental Table 1: Sample summary statistics, numeric variables. $\mathrm{n}=7016$.

\begin{tabular}{|c|c|}
\hline Sample Variable & Proportion \\
\hline Gender (Female) & $73.82 \%$ \\
Gender (Male) & $26.18 \%$ \\
\hline Country (France) & $92.63 \%$ \\
\hline Country (Switzerland) & $4.83 \%$ \\
\hline Country (Belgium) & $0.59 \%$ \\
\hline Country (Other) & $0.37 \%$ \\
\hline
\end{tabular}

Supplemental Table 2: Sample summary statistics, categorical variables. $\mathrm{n}=7016$.

\section{Note 2: Optimizing Sampling Approaches}

In this section we present in greater detail the results obtained when optimizing sampling based on specific times and days. To compare performance across time windows, we followed these steps: For a given individual and number of reports between 3 and 30, we 1) resampled without replacement and estimated affect dynamics measure 1000 times, 2) we 
applied a debiased step to each of these 1000 estimates, 3) we estimate an individual's Root Mean Square Error (RMSE) for each number of reports based on the full sample "true" estimates of affect dynamics measures, and 4) we average the RMSE across participants for each number of reports used in the computation of the affect dynamics measures. To debias the estimates, we first obtained a condition-specific bias by subtracting from the average of the population of true values of an affect dynamics measure, the average of the population of the same measure estimated with affect reports from our time-window of interest. We then subtracted this bias to each of our estimates of affect dynamics measures obtained from resampling 1000 times. For example, when analyzing the performance of the estimations of the TKEO with reports collected at night, we first obtained a condition-specific bias. To calculate this bias term, we 1) estimated each individual's TKEO in affect using all reports available, 2) estimated each individual's TKEO in affect using all reports collected at night, 3) averaged 1 and 2 across individuals, and 4) subtracted from the average of the population of TKEOs estimated with all reports, the average of the population of TKEOs estimated with the reports collected at night. This debiasing procedure allowed us to account for "condition fixed effects", any constant bias across individuals that did not affect the relative ordering of individuals in terms of their affect dynamics measure of interest. For completeness, we also present the results of the analyses without a debiasing step. Including the debiasing step did not substantially change our results. The results are presented in the supplemental figures 1-4. Supplemental figure 5 presents the results obtained when considering a sampling strategy that includes at least one observation from each time window. The estimations of performance in this last condition did not include a debiasing step. We followed the same procedure outlined here to compare performance when sampling across specific days. Supplemental figures 6-7 present the results obtained when sampling on specific days. 

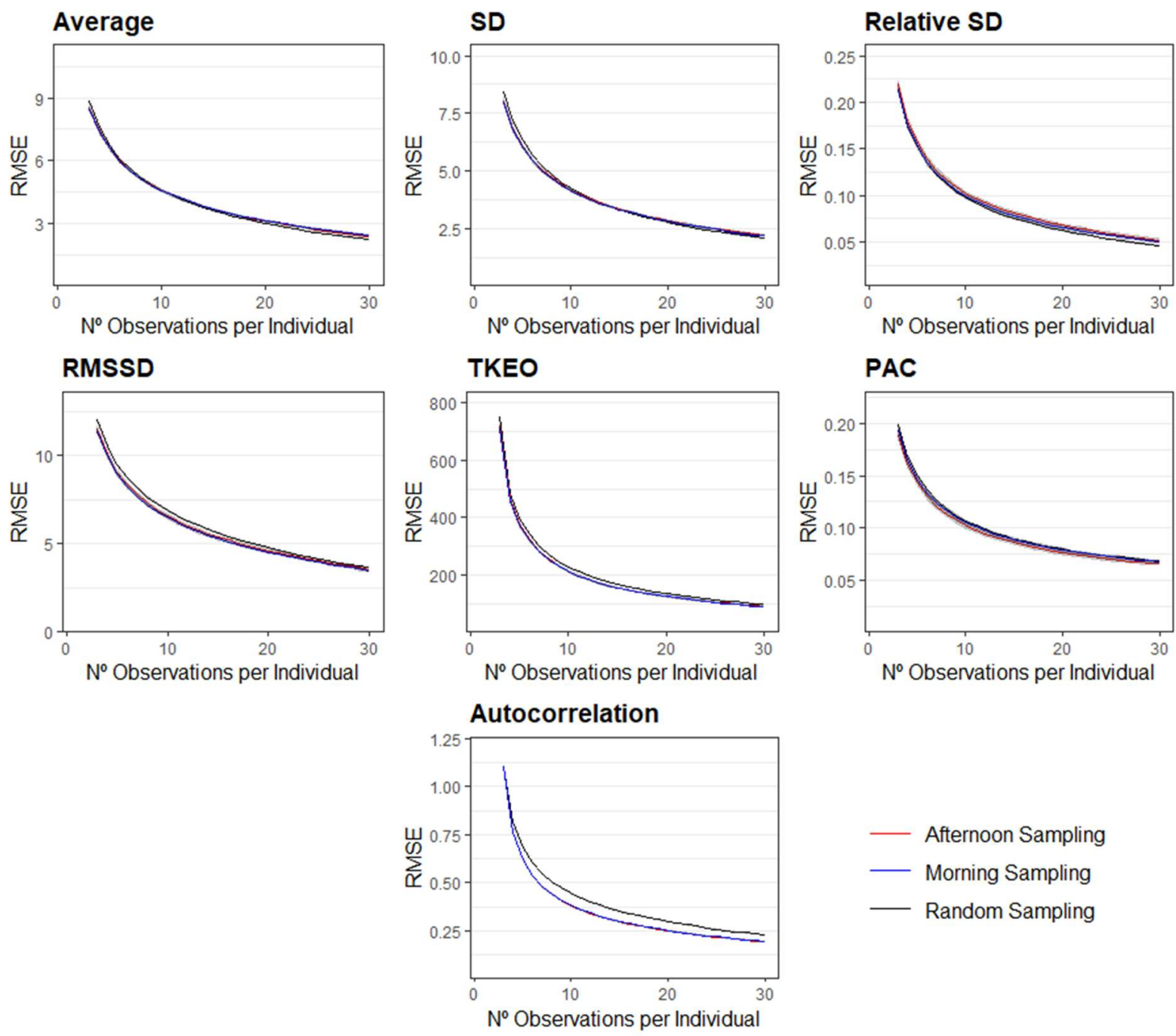

Figure S1. Between-subject mean RMSE for affect dynamics measures as a function of time of the day. Random times are depicted in black, morning (6am to noon) in blue, and afternoon (noon to $4 \mathrm{pm}$ ) in red. Gray areas around the lines represent the $95 \%$ confidence intervals for the average RMSE. These estimates are calculated after a debiasing step. 

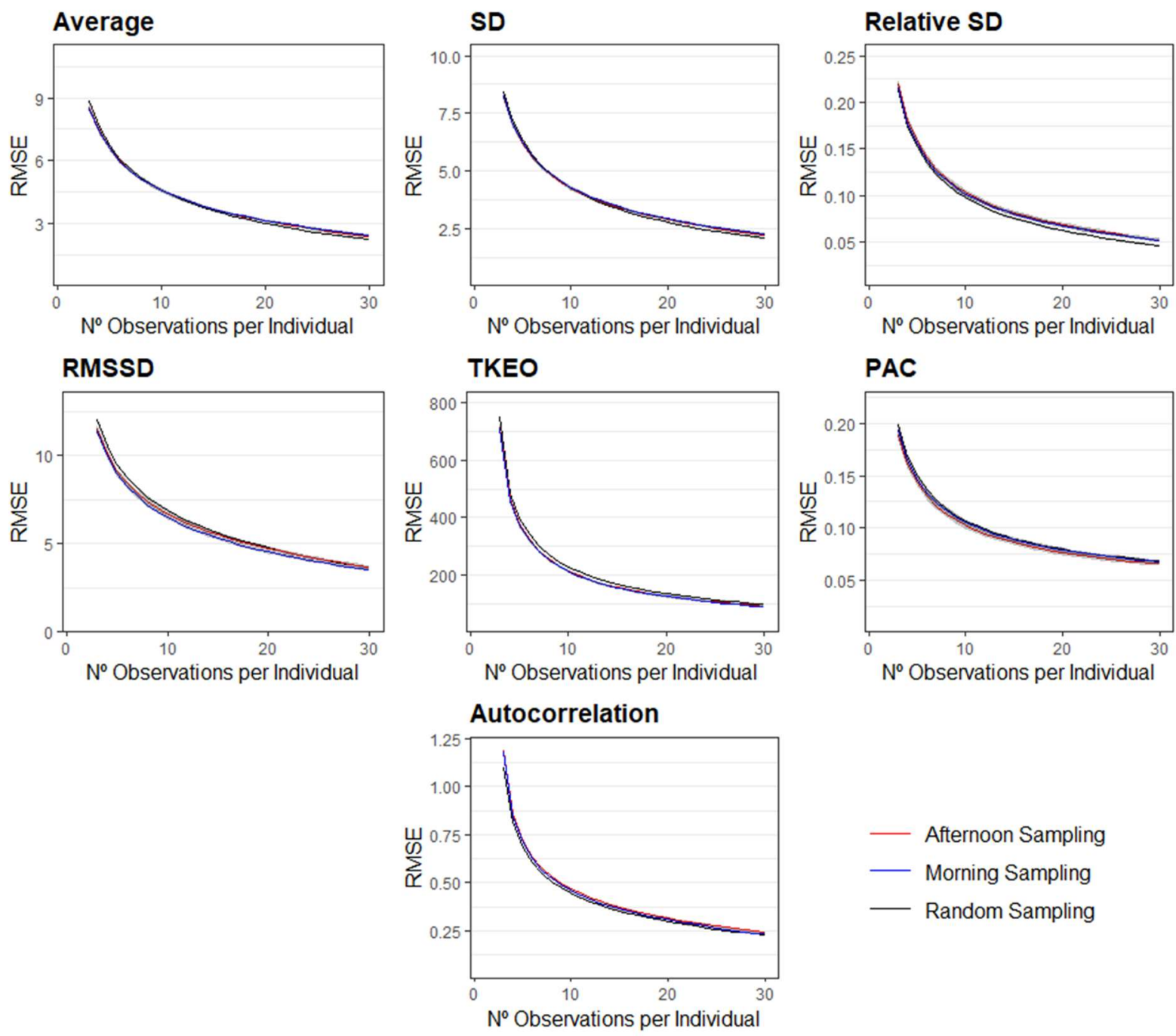

Figure S2. Between-subject mean RMSE for affect dynamics measures as a function of time of the day. Random times are depicted in black, morning (6am to noon) in blue, and afternoon (noon to $4 \mathrm{pm}$ ) in red. Gray areas around the lines represent the $95 \%$ confidence intervals for the average RMSE. These estimates are calculated without a debiasing step. 

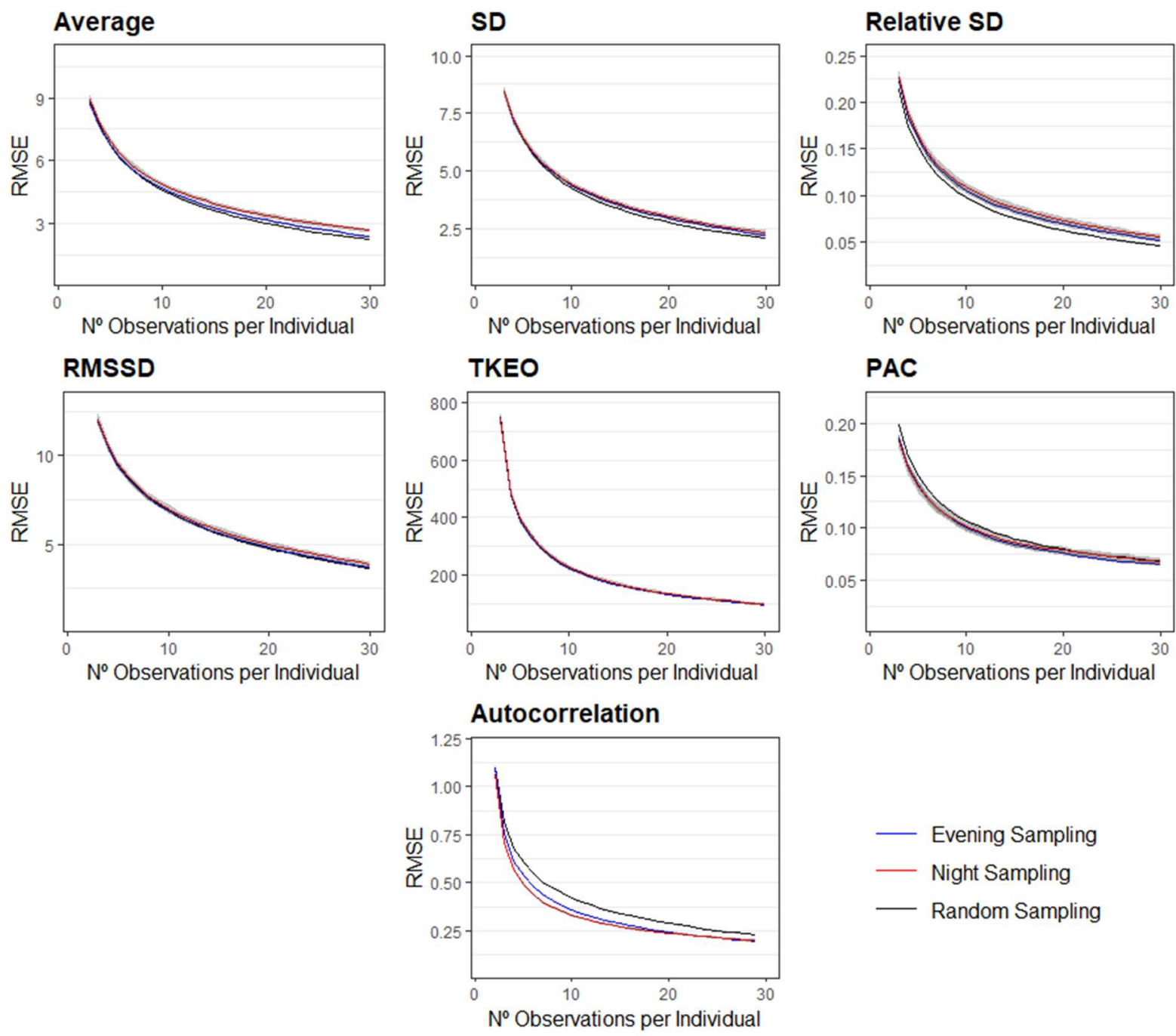

_ Evening Sampling
- Night Sampling
- Random Sampling

Figure S3. Between-subject mean RMSE for affect dynamics measures as a function of time of the day. Random times are depicted in black, evening (4pm to $8 \mathrm{pm})$ in blue, and night (8pm to 6am) in red. Gray areas around the lines represent the $95 \%$ confidence intervals for the average RMSE. These estimates are calculated after a debiasing step. 

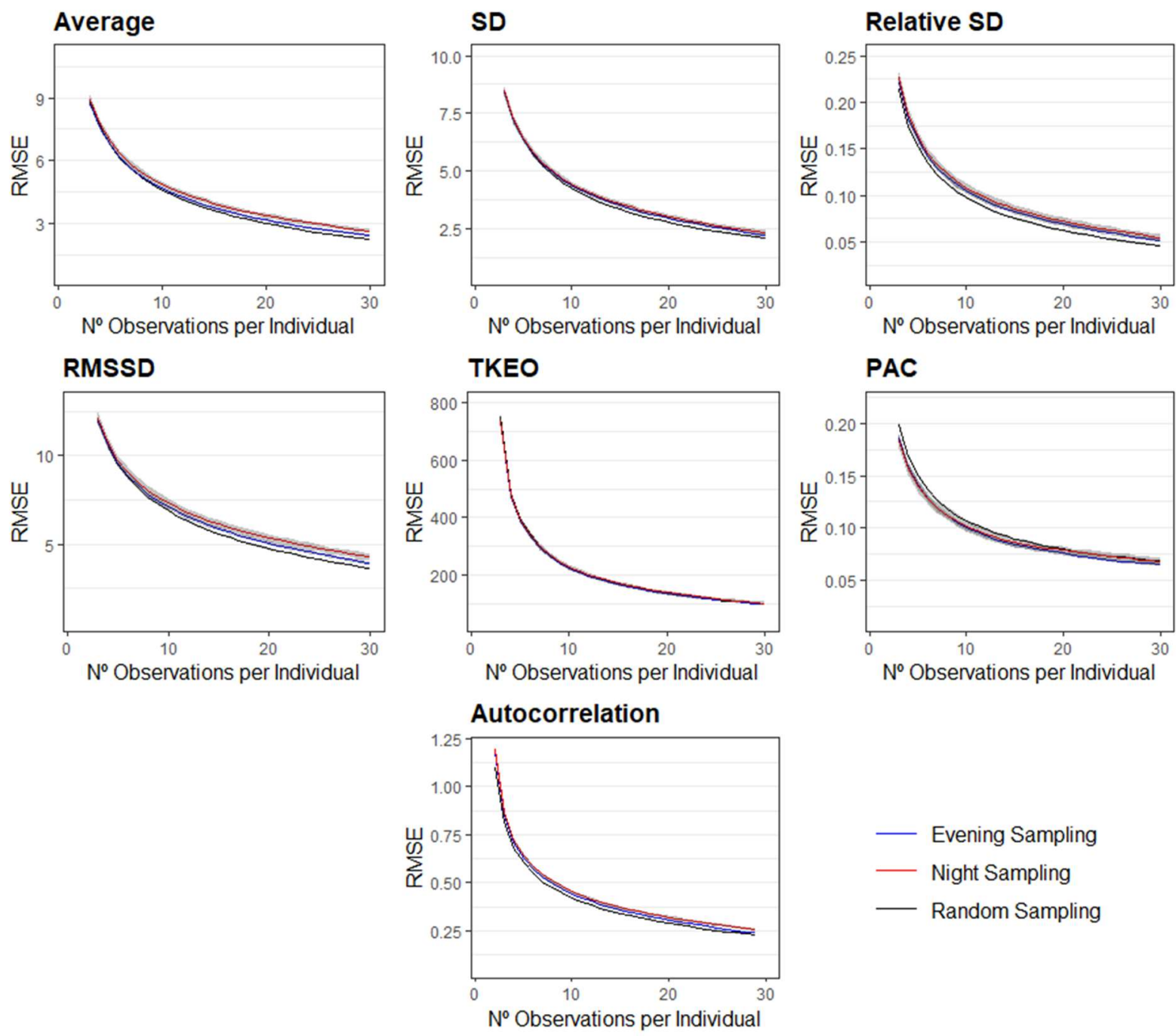

_ Evening Sampling
- Night Sampling
- Random Sampling

Figure S4. Between-subject mean RMSE for affect dynamics measures as a function of time of the day. Random times are depicted in black, evening (4pm to $8 \mathrm{pm}$ ) in blue, and night (8pm to 6am) in red. Gray areas around the lines represent the $95 \%$ confidence intervals for the average RMSE. These estimates are calculated without a debiasing step. 

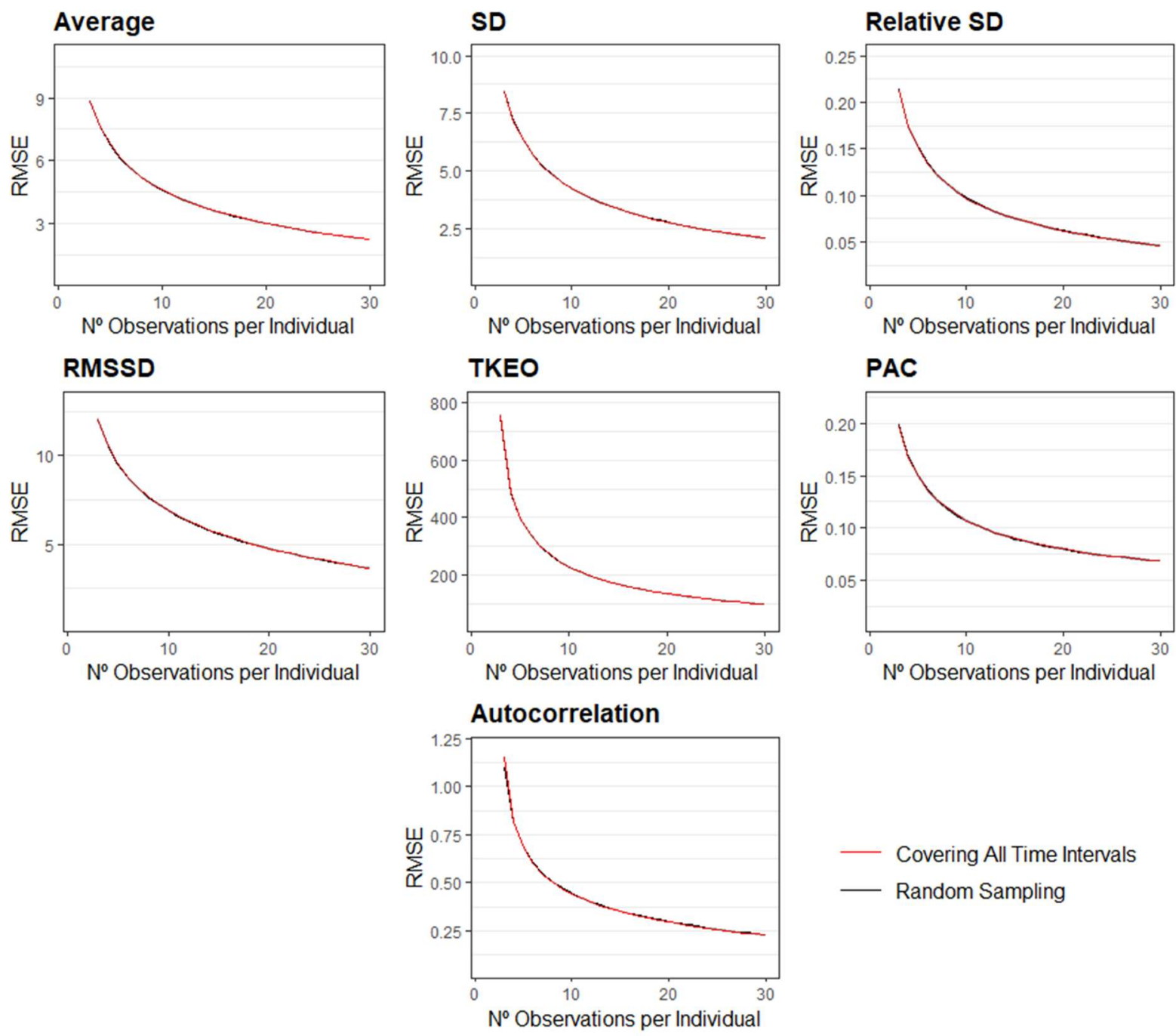

- Covering All Time Intervals

— Random Sampling

Figure S5. Between-subject mean RMSE for affect dynamics measures as a function of time of the day. Random times are depicted in black, and estimates obtained when sampling a minimum of 1 observation from each time interval (morning, afternoon, evening and night) in red. Gray areas around the lines represent the $95 \%$ confidence intervals for the average RMSE. 

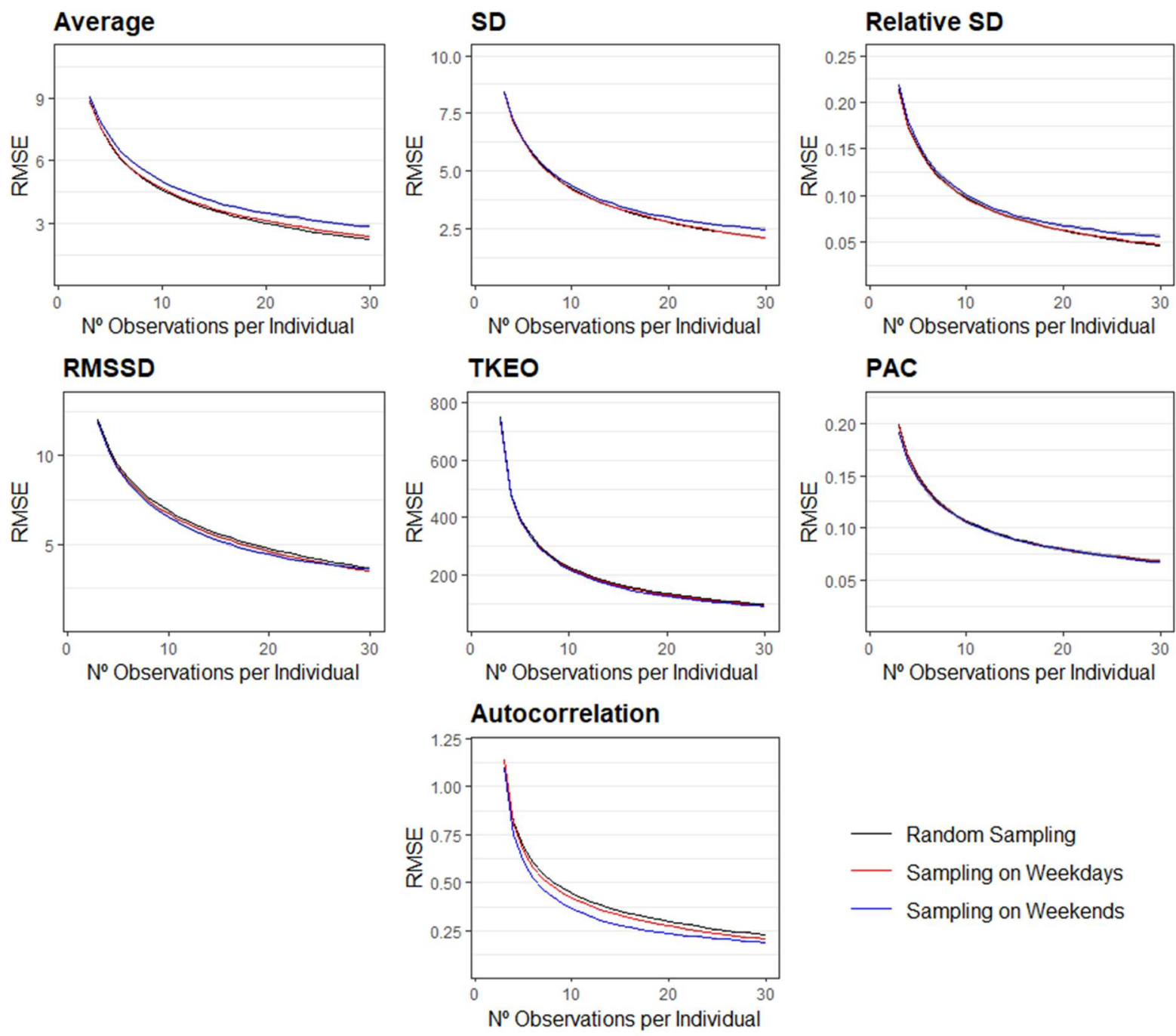

_ Random Sampling
- Sampling on Weekdays
- Sampling on Weekends

Figure S6. Between-subject mean RMSE for affect dynamics measures as a function of day of the week. Random days are depicted in black, weekends in blue, and weekdays (8pm to 6am) in red. Gray areas around the lines represent the $95 \%$ confidence intervals for the average RMSE. These estimates are calculated after a debiasing step. 

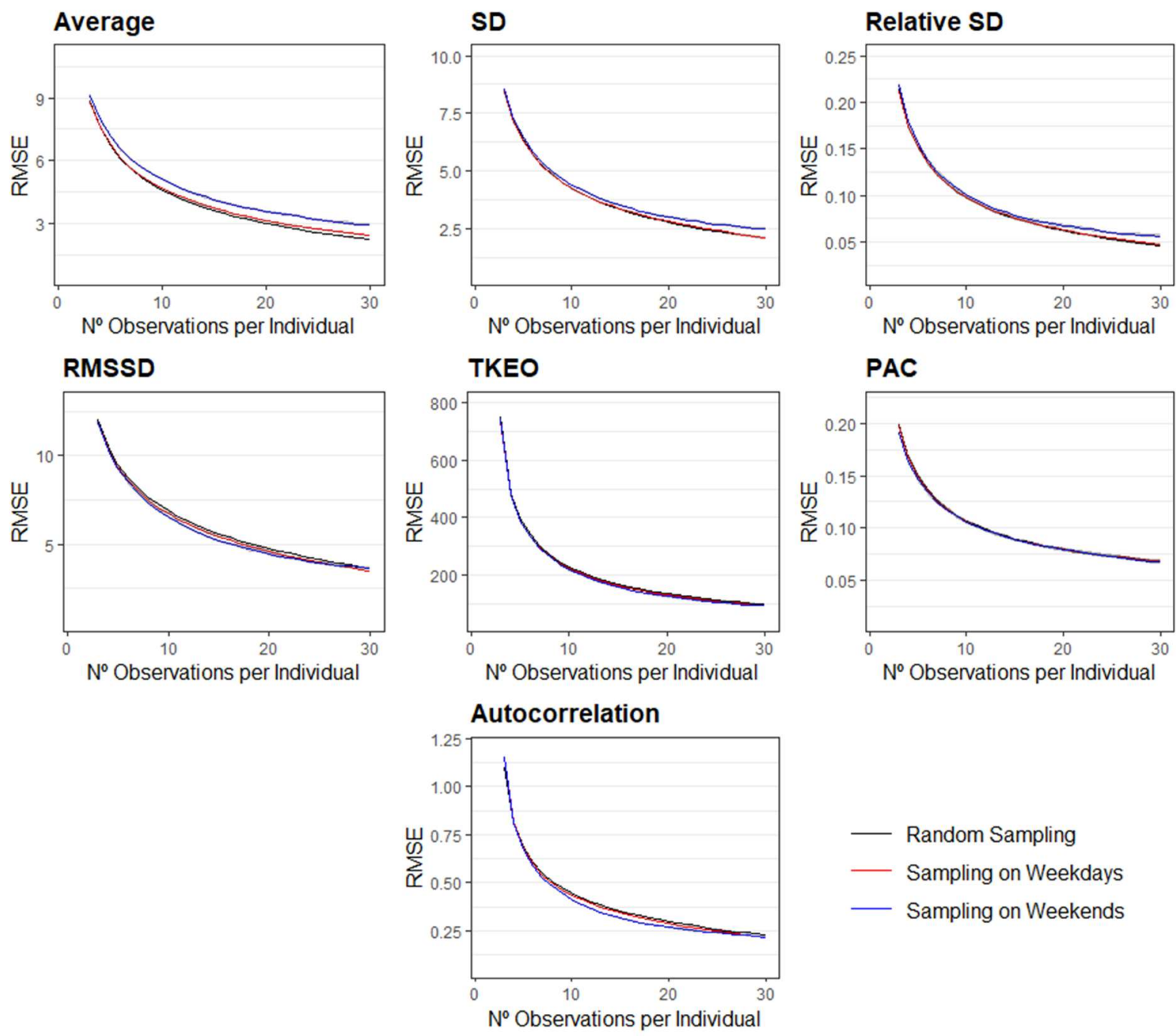

_ Random Sampling
- Sampling on Weekdays
- Sampling on Weekends

Figure S7. Between-subject mean RMSE for affect dynamics measures as a function of day of the week. Random times are depicted in black, weekends in blue, and weekdays (8pm to 6am) in red. Gray areas around the lines represent the $95 \%$ confidence intervals for the average RMSE. These estimates are calculated without a debiasing step. 


\section{Note 3: Statistical Power as a Function of Sampling}

In this note, we first detail the procedure followed to obtain Figure 4 of the main text and then present empirical power for all sampling combinations included in our study when interested in weak $(r=.10)$, medium-size $(r=.30)$ or strong correlations $(r=.50)$.

Figure 4 presents the minimum number of individuals and number of samples per individual for each affect dynamics measure to yield $80 \%$ power to detect a medium size relationship $(r=.30)$ with an alpha of 0.05 . To estimate the curves, we used the results of the empirical power estimations obtained in our main analyses (presented below in this Note). Using these results, for each affect dynamics measure, we first focused on those analyses that assumed an alpha of 0.05 and a medium size correlation $(r=.30)$. Then, for each number of samples per participant in our analyses (that is, from 5 to 50 in increments of 5), we selected the minimum number of participants that yielded a power larger than or equal to $80 \%$, and the maximum number of participants that yielded a power lower than or equal to $80 \%$. We divided the space between these two numbers of participants into a sequence with increments of 10 participants and linearly interpolated power for each number of participants included in this sequence. Using these interpolated power values, we selected, for each number of samples per participant, the minimum number of individuals with an approximated power larger than or equal to $80 \%$. This process yielded, for each affect dynamics measure, 10 combinations of individuals and number of samples per participants with an approximate power over $80 \%$. As small variations existed in power (approximated power ranged between 80\%-83\%) and we relied on linear approximations, we encountered some non-monotonic regions - that is, regions where the number of participants needed to achieve a minimum power of $80 \%$ did not decrease with the number of samples per participant but displayed minor increases. We directly imposed weak monotonicity by replacing these regions by sequences in which the number of participants remained unchanged as the number of samples 
per participant was increased. In doing so, for each affect dynamics measure we obtained our 10 final sampling combinations with an approximate power of $80 \%$. Using these 10 combinations, we regressed, for each affect dynamics measure, the logarithm of the number of participants on the number of samples per participant and its logarithm. This specification was selected based on fit - the average R-squared across affect dynamics measures was above $95 \%$. We used the fitted number of participants for each number of samples per participant to draw the curves. Supplemental Table 3 presents the final 10 sampling combinations used to run the regression models for each affect dynamics measure. We abstain from making power extrapolations, and therefore, for affect inertia ("Auto."), we do not provide the number of individuals needed when sampling affect 5 times from each participant. This number would be well above 5120 participants.

\begin{tabular}{cccccccc}
\hline Samples & \multicolumn{7}{c}{ Number of Individuals } \\
\hline & Average & SD & Rel. SD & RMSSD & TKEO & PAC & Auto. \\
& 120 & 230 & 260 & 300 & 1200 & 460 & - \\
10 & 110 & 150 & 160 & 200 & 360 & 260 & 1690 \\
15 & 110 & 130 & 140 & 160 & 240 & 210 & 810 \\
20 & 100 & 130 & 130 & 140 & 180 & 160 & 510 \\
25 & 100 & 120 & 120 & 140 & 170 & 160 & 400 \\
30 & 90 & 110 & 110 & 130 & 150 & 140 & 290 \\
35 & 90 & 110 & 110 & 120 & 140 & 140 & 250 \\
40 & 90 & 110 & 110 & 120 & 140 & 130 & 210 \\
50 & 90 & 110 & 110 & 120 & 130 & 130 & 190 \\
\hline 50 & 90 & 100 & 110 & 120 & 130 & 130 & 160 \\
\hline
\end{tabular}


Supplemental Table 3: Sampling combinations yielding an approximate power of $80 \%$ to detect a medium size association $(r=.30)$ with an alpha of 0.05 for each affect dynamics measure. "Samples" represent the number of samples per participant needed and "N.Ind" the total number of participants.

Next, we focused on the results of estimating the empirical power for weak $(r=.10)$ and strong correlations $(r=.50)$. To estimate power, we followed the same procedure presented in the main text. We took a conservative approach and set the type 1 error rate at 0.001 .
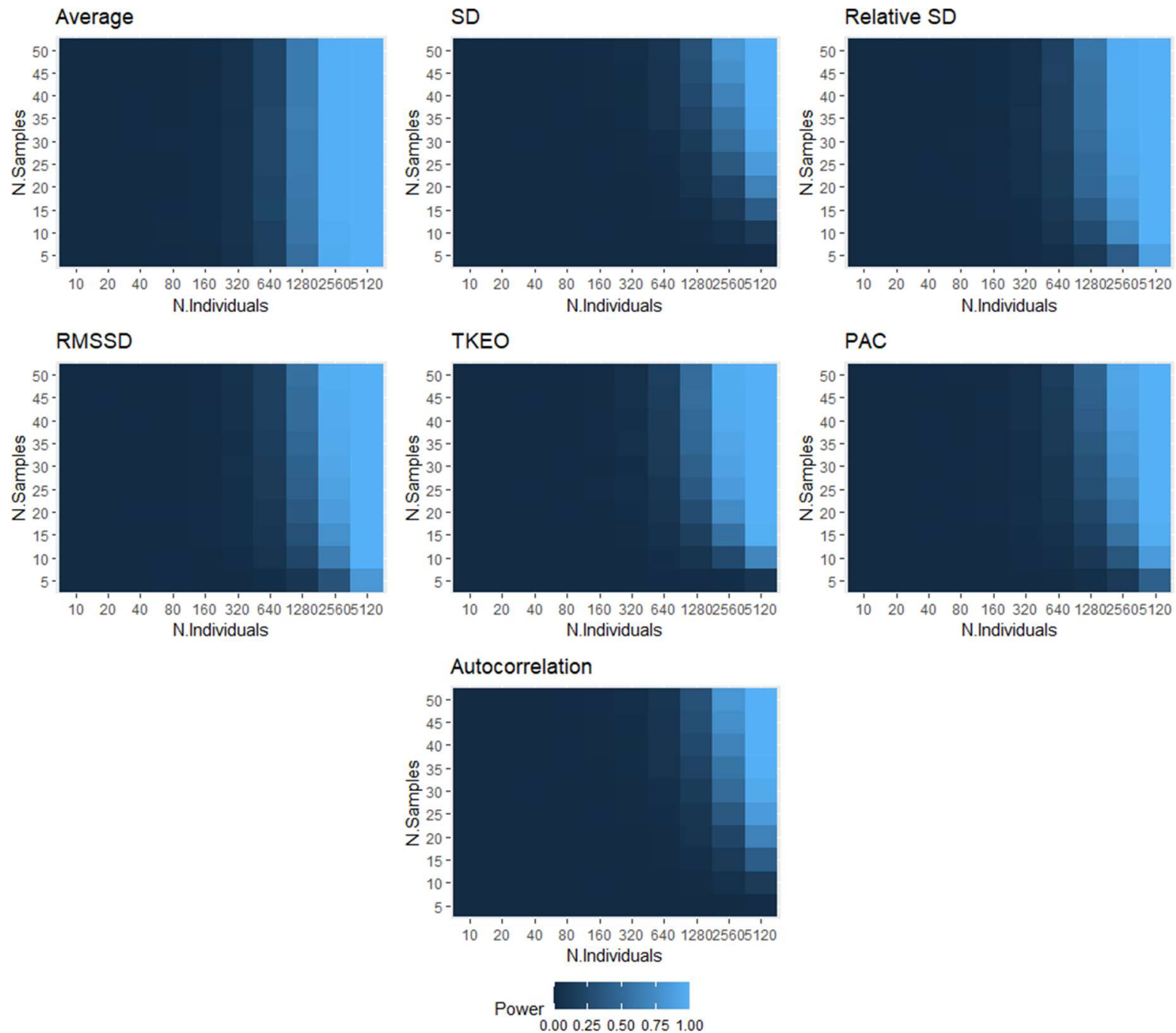
Supplemental Figure 8: Power as a function of sampling strategy. Each panel represents the power to detect a small correlation $(r=.10)$ between an affect dynamics measure and an outcome variable.
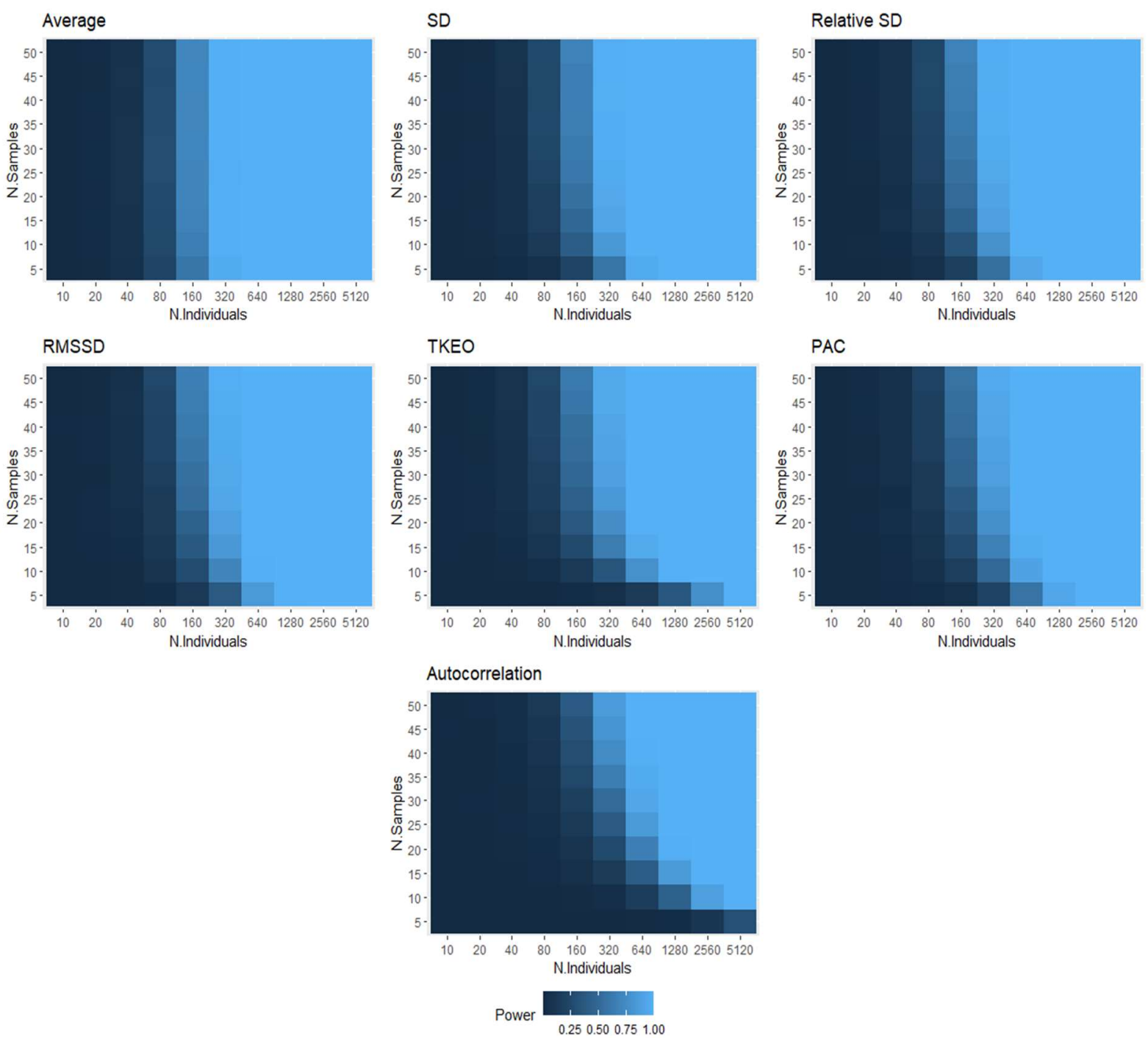

Supplemental Figure 9: Power as a function of sampling strategy. Each panel represents the power to detect a small correlation $(r=.30)$ between an affect dynamics measure and an outcome variable. 

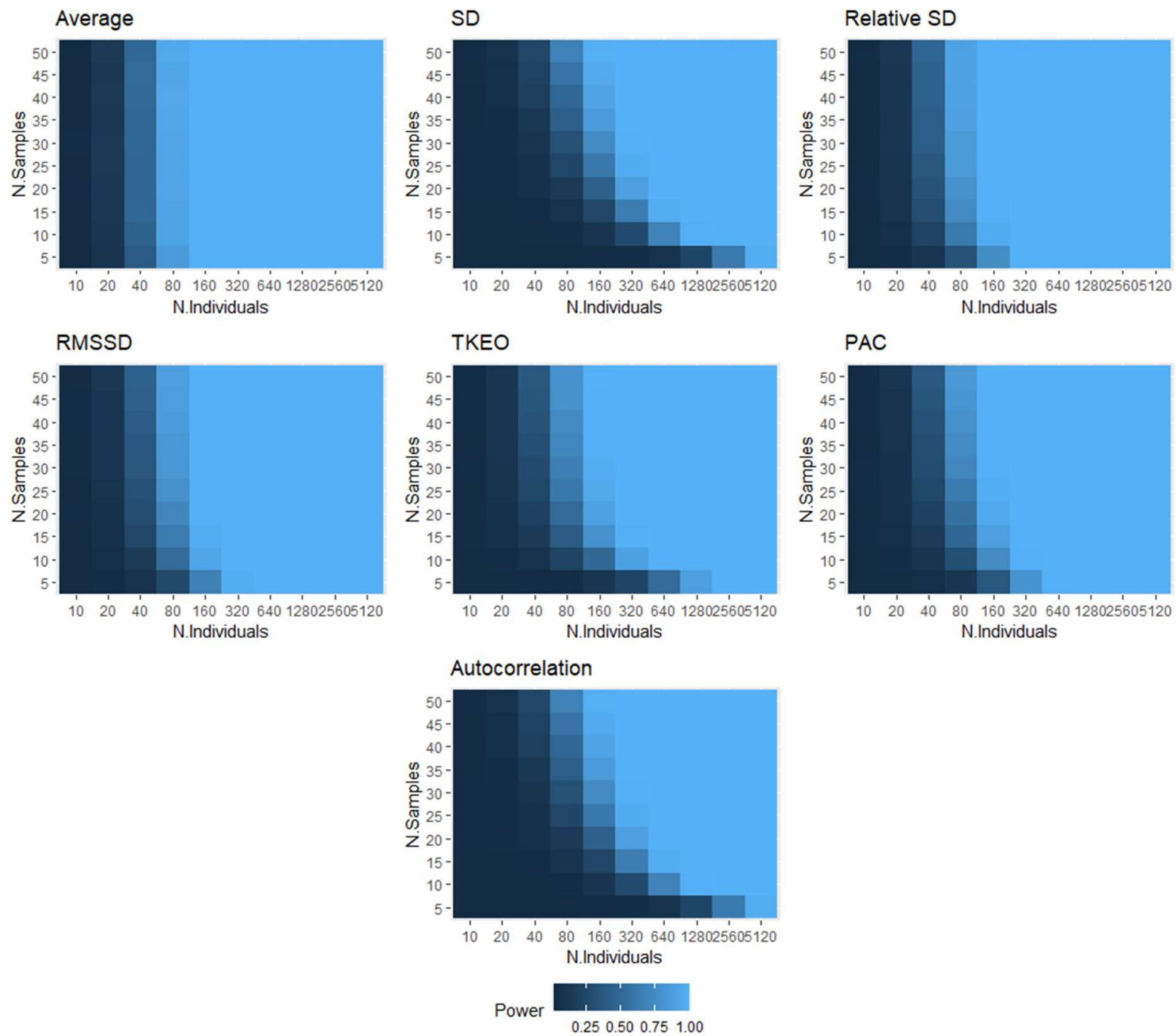

Supplemental Figure 10: Power as a function of sampling strategy. Each panel represents the power to detect a small correlation $(r=.50)$ between an affect dynamics measure and an outcome variable.

\section{Note 4: Average Effect of Number of Participants and Samples on Power}

In the following tables we present the results of regressing empirical power on the number of individuals and samples per participant for all affect dynamics measures. Note our sample size for each measure is 1500 observations as we include power obtained from our 100 sampling combinations, 3 effect sizes (small, medium and large) and 5 alpha levels $(0.1,0.05$, $0.01,0.005,0.001)$. 


\begin{tabular}{lcccc} 
& $\begin{array}{c}\text { Power } \\
\text { (All Measures) }\end{array}$ & $\begin{array}{c}\text { Power } \\
\text { (Average) }\end{array}$ & $\begin{array}{c}\text { Power } \\
(\text { SD })\end{array}$ & $\begin{array}{c}\text { Power } \\
(\text { Rel.SD })\end{array}$ \\
\hline $\log ($ Individuals $)$ & $0.156^{* * *}$ & $0.153^{* * *}$ & $0.157^{* * *}$ & $0.157^{* * *}$ \\
& $(0.001)$ & $(0.003)$ & $(0.003)$ & $(0.003)$ \\
$\log ($ Samples $)$ & $0.080^{* * *}$ & 0.008 & $0.045^{* * *}$ & $0.048^{* * *}$ \\
& $(0.004)$ & $(0.010)$ & $(0.010)$ & $(0.010)$ \\
Constant & $-0.525 * * *$ & $-0.211^{* * *}$ & $-0.379 * * *$ & $-0.394 * * *$ \\
& $(0.014)$ & $(0.037)$ & $(0.037)$ & $(0.037)$ \\
\hline Observations & 10500 & 1500 & 1500 & 1500 \\
\hline & 0.567 & 0.562 & 0.583 & 0.585 \\
\hline
\end{tabular}

Supplemental Table 4: OLS estimates of the effect of number of individuals and samples per participant on statistical power across effect sizes and significance levels. Standard errors are in parentheses. Statistical significance: ${ }^{*} \mathrm{p}<0.1 ; *{ }^{*} \mathrm{p}<0.05 ; * * * p<0.01$ 


\begin{tabular}{|c|c|c|c|c|}
\hline & $\begin{array}{c}\text { Power } \\
\text { (RMSSD) }\end{array}$ & $\begin{array}{l}\text { Power } \\
\text { (TKEO) }\end{array}$ & $\begin{array}{l}\text { Power } \\
\text { (PAC) }\end{array}$ & $\begin{array}{l}\text { Power } \\
\text { (Auto.) }\end{array}$ \\
\hline \multirow[t]{2}{*}{$\log$ (Individuals) } & $0.160 * * *$ & $0.158 * * *$ & $0.160 * * *$ & $0.147 * * *$ \\
\hline & $(0.003)$ & $(0.003)$ & $(0.003)$ & $(0.003)$ \\
\hline \multirow[t]{2}{*}{$\log ($ Samples $)$} & $0.058 * * *$ & $0.128 * * *$ & $0.079 * * *$ & $0.196^{* * *}$ \\
\hline & $(0.010)$ & $(0.010)$ & $(0.010)$ & $(0.010)$ \\
\hline \multirow[t]{2}{*}{ Constant } & $-0.454 * * *$ & $-0.709 * * *$ & $-0.551 * * *$ & $-0.974 * * *$ \\
\hline & $(0.036)$ & $(0.036)$ & $(0.037)$ & $(0.036)$ \\
\hline Observations & 1500 & 1500 & 1500 & 1500 \\
\hline $\mathrm{R} 2$ & 0.596 & 0.612 & 0.596 & 0.598 \\
\hline
\end{tabular}

Supplemental Table 5: OLS estimates of the effect of number of individuals and samples per participant on statistical power across effect sizes and significance levels. Standard errors are in parentheses. Statistical significance: ${ }^{*} \mathrm{p}<0.1 ; *{ }^{*} \mathrm{p}<0.05 ; * * * \mathrm{p}<0.01$

\section{Note 5: Plausible Effect Sizes}

We collected our data using "58 seconds", a free smartphone application. At sign-up, the participants answered a few questions regarding demographic information. Using such information, we coded the variable "Gender" to take a value of 1 for male participants and 0 for female participants. The numeric variable "Age" represents a participant's age in years. Data on life meaning, satisfaction, sleep and proportion of time spent with different groups of people or engaging in different activities was collected using a system of random notifications. Participants using the app received questionnaire prompts at random times of the day. These questionnaires consisted of 4 to 6 questions from a large battery of items. For 
meaning in life, the participants were asked to rate the following statement from 0 ("Not at all”) to 100 (“Absolutely”): "Here and now I feel like I'm living a meaningful life”. Similarly, for life satisfaction, participants provide a rating from 0 (Dissatisfied with my life) to 100 (Satisfied with my life) to the following statement: "Here and now, I feel...". For life meaning as for life satisfaction, we used these numeric reports to estimate the seven dynamic measures included in this paper (Average, SD, Rel. SD, RMSSD, TKEO, PAC and Autocorrelation). Sleep was measured by asking participants the amount of sleep hours they had last night. Participants provided a numeric report ranging from 0 to 15 , and we averaged across sleep reports to obtain an individual's average hours of sleep. Finally, some questionnaires included a list of activities and a list of groups of people. The participants facing these lists were asked to select all activities that they were doing before answering the questionnaire and select all the groups of people with whom they were when answering the questionnaire. For simplicity, we restricted our attention to the proportion of time an individual spent with family, friends, alone, studying or working, and exercising. To obtain proxies for the amount of time that an individual spent with these people or doing these activities, we estimated the proportion of times (out of all times that the participant was presented with each list) that the user reported being with the specific group of people or doing a specific activity.

For each participant, aside from these variables, we estimated the seven affect dynamics measures included in our main analyses using the full sample of affect reports at our disposal. To provide the reader with effect sizes to serve as reference, we estimated the Pearson's r coefficient between each affect dynamics measure and the demographic, wellbeing and time allocation variables described in the previous paragraph. The resulting coefficients are presented in the Supplemental Table 6. 


\begin{tabular}{|c|c|c|c|c|c|c|c|}
\hline Outcome & Average & SD & Rel. SD & RMSSD & TKEO & PAC & Auto. \\
\hline Age & 0.059 & -0.248 & -0.191 & -0.282 & -0.247 & -0.269 & 0.093 \\
\hline Gender & 0.053 & -0.098 & -0.047 & -0.093 & -0.081 & -0.085 & -0.012 \\
\hline $\begin{array}{c}\text { Average Meaning in } \\
\text { life }\end{array}$ & 0.839 & -0.231 & 0.096 & -0.201 & -0.194 & -0.236 & -0.029 \\
\hline SD Meaning in life & 0.019 & 0.520 & 0.431 & 0.468 & 0.452 & 0.413 & 0.055 \\
\hline $\begin{array}{l}\text { Rel.SD Meaning in } \\
\text { life }\end{array}$ & 0.144 & 0.414 & 0.623 & 0.365 & 0.373 & 0.318 & 0.059 \\
\hline $\begin{array}{c}\text { RMSSD Meaning in } \\
\text { life }\end{array}$ & 0.014 & 0.472 & 0.387 & 0.469 & 0.448 & 0.422 & -0.031 \\
\hline TKEO Meaning in life & 0.039 & 0.295 & 0.280 & 0.282 & 0.284 & 0.249 & 0.005 \\
\hline PAC Meaning in life & 0.040 & 0.381 & 0.355 & 0.387 & 0.383 & 0.360 & -0.041 \\
\hline Auto. Meaning in life & 0.017 & 0.016 & 0.026 & -0.076 & -0.058 & -0.082 & 0.199 \\
\hline $\begin{array}{l}\text { Average Life } \\
\text { satisfaction }\end{array}$ & 0.856 & -0.197 & 0.109 & -0.144 & -0.144 & -0.181 & -0.080 \\
\hline SD Life satisfaction & -0.076 & 0.508 & 0.393 & 0.439 & 0.428 & 0.408 & 0.096 \\
\hline $\begin{array}{l}\text { Rel.SD Life } \\
\text { satisfaction }\end{array}$ & 0.141 & 0.363 & 0.579 & 0.323 & 0.329 & 0.286 & 0.053 \\
\hline $\begin{array}{l}\text { RMSSD Life } \\
\text { satisfaction }\end{array}$ & -0.087 & 0.471 & 0.353 & 0.447 & 0.431 & 0.420 & 0.013 \\
\hline $\begin{array}{l}\text { TKEO Life } \\
\text { satisfaction }\end{array}$ & 0.003 & 0.251 & 0.227 & 0.231 & 0.241 & 0.208 & 0.021 \\
\hline PAC Life satisfaction & -0.034 & 0.375 & 0.325 & 0.366 & 0.364 & 0.349 & -0.014 \\
\hline Auto. Life satisfaction & 0.054 & -0.007 & 0.029 & -0.080 & -0.061 & -0.079 & 0.174 \\
\hline Average sleep & 0.138 & 0.001 & 0.028 & 0.036 & 0.023 & 0.030 & -0.065 \\
\hline Time spent alone & -0.239 & 0.033 & -0.026 & 0.002 & 0.015 & 0.009 & 0.064 \\
\hline $\begin{array}{l}\text { Time spent with } \\
\text { family }\end{array}$ & 0.072 & 0.001 & 0.030 & 0.008 & 0.004 & 0.001 & -0.012 \\
\hline
\end{tabular}


Time spent with friends 0.064

0.100

0.113

0.105

0.091

0.106

$-0.025$

Time spent at work/studying

$$
0.040
$$

$-0.033-0.027$

$-0.029$

$-0.031$

$-0.023$

Time spent exercising

$$
0.092
$$

$-0.018$

0.016

$-0.015$

$-0.017$

$-0.017$

$-0.006$

Supplemental Table 6: Effect sizes (Pearson's $r$ ) of the correlation between different outcomes and measures of affect. Outcomes correspond to demographic variables, variables estimating the propensity of individuals to perform an activity or being in the presence of others or measures of life satisfaction, meaning and sleep. The correlations were estimated using our full sample of 7016 individuals each providing a minimum of 50 affect reports.

\section{Note 6: Statistical power in the literature}

We reviewed the sampling characteristics of 423 papers ambulatory assessment studies of affect included in five major review articles (Aan het Rot et al., 2012; Dunster et al., 2021; Ebner-Priemer \& Trull, 2009; Houben et al., 2015; Myin-Germeys et al., 2009) and identified 88 papers that estimated at least one core measure of affect dynamics. Using our R-package ("powerAD”, https://sergiopirla.github.io/powerAD), we analyzed the statistical power achieved in each of these studies. Note that for 39 of the 88 included studies, we could not get a precise power estimation but an approximate one, as the number of affect reports per participant collected in these studies were over 50. For those studies, we approximated power by censoring the number of samples per participant at 50 . We do not expect this censoring to have large effects on our results as 1) most of the numbers of reports are close to 50 (in fact, almost half of the studies that collect more than 50 samples per participant also collect less than 60 samples per participant, see Figure 1) and, 2) our power estimations presented in 
Figure 4 suggests that power is a concave function of the number of affect reports per individual.

\begin{tabular}{ccccc}
\hline Affect Measure & $r=.10$ & $r=.30$ & $r=.50$ & Publications $(N)$ \\
\hline Average & $1 \%$ & $32 \%$ & $84 \%$ & 81 \\
SD & $2 \%$ & $30 \%$ & $82 \%$ & 50 \\
RMSSD & $0 \%$ & $16 \%$ & $74 \%$ & 19 \\
TKEO & $0 \%$ & $0 \%$ & $100 \%$ & 1 \\
PAC & $0 \%$ & $0 \%$ & $100 \%$ & 2 \\
AUTO & $0 \%$ & $10 \%$ & $70 \%$ & 10 \\
\hline
\end{tabular}

Supplemental Table 7: Proportion of experience sampling studies of affect dynamics that achieve an $80 \%$ statistical power to detect an effect of size $r=\{0.1,0.3,0.5\}$ with a $95 \%$ confidence level.

Using a similar procedure, we also estimated the average power in the literature for different effect sizes (assuming an alpha level of 0.05). 


\begin{tabular}{ccccc}
\hline Affect Measure & $\begin{array}{c}\text { Power to } \\
\text { detect an } \\
\text { effect with } \\
r=.10\end{array}$ & $\begin{array}{c}\text { Power to } \\
\text { detect an } \\
\text { effect with } \\
r=.30\end{array}$ & $\begin{array}{c}\text { Power to } \\
\text { detect an } \\
\text { effect with } \\
r=.50\end{array}$ & Publications $(N)$ \\
\hline Average & $16 \%$ & $65 \%$ & $92 \%$ & 81 \\
SD & $16 \%$ & $64 \%$ & $90 \%$ & 50 \\
RMSSD & $13 \%$ & $60 \%$ & $88 \%$ & 19 \\
TKEO & $11 \%$ & $49 \%$ & $86 \%$ & 1 \\
PAC & $11 \%$ & $62 \%$ & $94 \%$ & 10 \\
\hline AUTO & $10 \%$ & $46 \%$ & $80 \%$ & 2 \\
\hline
\end{tabular}

Supplemental Table 8: Average power of experience sampling studies of affect dynamics for an effect of size $r=\{0.1,0.3,0.5\}$ with a $95 \%$ confidence level. 